\title{
Extracting Einstein from the loop-level double-copy
}

\author{
John Joseph M. Carrasco ${ }^{a, b}$ and Ingrid A. Vazquez-Holm ${ }^{b}$ \\ ${ }^{a}$ Department of Physics $\&$ Astronomy, Northwestern University, \\ Evanston, Illinois 60208, U.S.A. \\ ${ }^{b}$ Institut de Physique Théorique, CEA Saclay, \\ Orme des Merisiers, Gif-sur-Yvette, France \\ E-mail: john-joseph.carrasco@ipht.fr, ingrid.holm@physics.uu.se
}

\begin{abstract}
The naive double-copy of (multi) loop amplitudes involving massive matter coupled to gauge theories will generically produce amplitudes in a gravitational theory that contains additional contributions from propagating antisymmetric tensor and dilaton states even at tree-level. We present a graph-based approach that combines the method of maximal cuts with double-copy construction to offer a systematic framework to isolate the pure Einstein-Hilbert gravitational contributions through loop level. Indeed this allows for a bootstrap of pure-gravitational results from the double-copy of massive scalar-QCD. We apply this to construct the novel result of the $D$-dimensional one-loop five-point QFT integrand relevant in the classical limit to generating observables associated with the radiative effects of massive black-hole scattering via pure Einstein-Hilbert gravity.
\end{abstract}

Keywords: Black Holes, Duality in Gauge Field Theories, Scattering Amplitudes

ArXiv EPrint: 2108.06798 


\section{Contents}

1 Introduction 1

2 Review $\quad 4$

2.1 Gravity amplitudes from the double copy 4

2.2 Method of maximal cuts 5

3 Projective double-copy $\quad 6$

4 Extracting Einstein-Hilbert at tree and loop level $\quad 7$

4.1 Tree-level 8

$\begin{array}{lll}4.2 & \text { Loop level } & 11\end{array}$

$\begin{array}{lll}4.2 .1 & \text { Four-point one-loop } & 13\end{array}$

$\begin{array}{lll}4.2 .2 & \text { Five-point one-loop } & 18\end{array}$

5 Conclusion $\quad 20$

\section{Introduction}

The color-dual double copy construction $[1,2]$ of quantum field theory predictions can be used to build scattering amplitudes for a wide variety of theories from phenomenological effective field theories to formal completions of Yang-Mills and Gravity like open and closed string theories. ${ }^{1}$ The first concrete hint of this web of theories was the construction of closed string tree-level amplitudes from sums over permutations of Chan-Paton-stripped open-string amplitudes via the celebrated relations of Kawai, Lewellen and Tye [4] (KLT). In the low energy limit these relations allow the expression of tree-level graviton amplitudes in terms of sums over permutations of ordered tree-level Yang-Mills amplitudes. In the color-dual approach of Bern, Johansson, and one of the current authors (BCJ) the building blocks of gravitational predictions are gauge theory kinematic graph weights that satisfy the duality between color and kinematics. This means that the gauge theory predictions can be expressed in a representation where, graph-by-graph, kinematic weights obey the same algebraic relations as the color weights. The gravitational double copy prediction is then obtained by exchanging the gauge theory's color factors for a second set of kinematic weights. This approach can be used to generate the field-theory limit of KLT relations at tree-level [1], by inverting the relationship between color-dual numerators and ordered-amplitudes, and generalizes to integrand representations at multiloop levels [2, 5]. The theory of massive scalars minimally coupled to Yang-Mills, massive scalar-QCD, is

\footnotetext{
${ }^{1}$ See, e.g., section 5 of ref. [3] for a recent review of the color-dual web of theories.
} 
compatible with this duality both at tree level $[6,7]$, as well as at the one-loop integrand level [8].

The technical simplicity of color-dual double-copy relating quantum gauge and gravitational predictions, followed closely by the advent of precision gravitational wave science, has invited a renewed interest in applying quantum insights to classical predictions. One natural path [9] is to first calculate the coefficients of higher derivative operators in a classical effective field theory by lining up its amplitudes with amplitudes calculated in the more UV complete theory (i.e. Einstein-Hilbert gravity), then to calculate the classical observables in the simpler EFT. Careful construction of classical gauge-invariant observables have also allowed construction directly from the quantum field theory observables themselves [10, 11]. There are an increasing number of classical (and semi-classical) field theory solutions, both perturbative and non-perturbative, guided by quantum color-dual double-copy, ${ }^{2}$ including quantum double-copies against a classically double-copied background $[41,42]$. Critically, amplitude calculation of novel precision observables relevant to gravitational-wave physics have been achieved using both EFT and direct approaches [4350]. Indeed, the highest precision post-Minkowskian $\mathcal{O}\left(G^{4}\right)$ correction to the scattering of classical non-rotating black holes has involved a synthesis of effective field theory techniques, advanced multiloop integration innovation, and double-copy applied to tree-level scattering amplitudes [47]. Scattering amplitudes in massive scalar-QCD, where massive scalars are coupled via Yang-Mills, can be used via double-copy to construct the scattering of massive scalars coupled to gravitons, whose classical limit describes the evolution and interaction of non-rotating black holes. At tree level, color-dual amplitudes in massive scalar QCD have been presented for up to six-points with three pairs of massive scalars [7], and in ref. [8] it was conjectured that a simple bootstrap extends to all multiplicity and loop-level using only color-kinematics and factorization, verifying through one-loop five-point.

The straightforward double copy of Yang-Mills scattering without any particular state management results in predictions of the so called $\mathcal{N}=0$ supergravity, where - in addition to gravitons - a massless scalar (often called a dilaton) and an anti-symmetric two-form (dual to an axion in four dimensions) can propagate. These contributions can be avoided for external gravitons at tree-level for massless theories by coordinating the external states of gluons, but become relevant at loop level. Such extra-state contributions affect even treelevel when gravitons are coupled to massive matter [7, 51, 52]. Such long-range mediators can survive the classical limit, and so must be projected out or otherwise removed if one is targeting classical predictions equivalent to that of pure Einstein-Hilbert gravity. There can be additional differences in double-copy amplitudes, such as additional local contact terms only between massive states. Such massive-state local contact terms are irrelevant to long range classical physics and so will not concern us here.

There are many strategies for removing the extra states arising from naive doublecopy. The first examples of gravitational multi-loop cut-construction involved tree-level cuts built from ordered gauge-theory cuts by applying field theory KLT relations (see, e.g. [53]). Cut construction for pure-gravity theories can progress by explicitly subtracting

\footnotetext{
${ }^{2}$ See, e.g., refs. [12-40] for a fascinating, but by no means complete, set of examples.
} 
out contributions from unwanted states (cf. refs. [54, 55]). At loop level, the classical limit of amplitude integrands for binary black holes has been calculated up to three-loops using generalized unitarity cuts and the double copy of tree-level amplitudes to construct pure-graviton integrands [47] via the method of maximal cuts [56].

The method of maximal cuts [56] offers a hierarchical approach to perturbative quantization. Here we combine it with double-copy construction at the off-shell integrand level. Thus we construct pure Einstein-Hilbert gravity integrands by starting with compact expressions for the $\mathcal{N}=0$ supergravity integrands and systematically projecting out any non-gravitational propagating modes. We call this approach the "projective double copy", and demonstrate here that it offers a systematic isolation of local gravitational contributions.

Of course, if one is only interested in classical gravitation, it is not necessary to build consistent loop-level integrands at all, not to mention the generation of off-shell $\mathcal{N}=0$ supergravity integrands. One can employ (cf. refs. [43, 44, 47]) a variant of the method of maximal cuts to directly target only the relevant classical contributions to Einstein-Hilbert gravity. These efficient approaches never construct a color-dual integrand on the gauge side, nor build a complete quantum integrand on the gravity side. Rather, they double-copy at tree-level and apply unitarity methods to build the classically relevant Einstein-Hilbert integrands, minimizing any cut information beyond what is required for the known classical contributions. If we aspire, however, to apprehend the connections between theories in the web of theories at the integrand level, it is natural to develop techniques to map out and relate graph by graph the relevant integrands of both $\mathcal{N}=0$ supergravity as well as Einstein-Hilbert as we present here. Here we double-copy entire quantum integrands directly at the loop level, and then use generalized unitarity systematically to project out all unwanted states, hierarchically constructing the quantum difference integrands between $\mathcal{N}=0$ supergravity and Einstein-Hilbert gravity.

It is a good question as to whether it is possible to double-copy directly to puregravity integrands. In the massless case Johansson and Ochirov introduced [57] a prescription involving ghostly matter to remove unwanted states at loop-level. Inspired by this approach, ref. [58] constructed Einstein-Hilbert gravitation amplitudes involving massive matter through five-points at tree level by introducing a massless, ghostly scalar in the gauge theory. A side benefit of our approach is the generation of difference-integrand data which may clarify the path forward at loop-level involving massive matter. This data provides loop-level double-copy targets for identifying the appropriate state-ghost contributions arising from color-dual integrands involving massive states.

We will review the color-kinematics duality and the method of maximal cuts in section 2. We introduce our approach to projective double-copy construction via the method of maximal cuts in section 3 . In section 4 we will demonstrate our approach first at tree-level in section 4.1, then the one-loop correction to two distinct-mass scalar particles scattering gravitationally in section 4.2.1, as well as including gravitational radiation in the related five-point one-loop amplitude integrand in section 4.2.2. We conclude and discuss potential next steps in section 5 . 


\section{Review}

\subsection{Gravity amplitudes from the double copy}

The general form of a $m$-point $L$-loop gauge theory amplitude $\mathcal{A}_{m}^{(L)}$ in $D$ space-time dimensions with gluons and massive particles can be written as

$$
\mathcal{A}_{m}^{(L)}=i^{L} g^{m-2+2 L} \sum_{i \in \Gamma} \int \prod_{l=1}^{L} \frac{d^{D} p_{l}}{(2 \pi)^{D}} \frac{1}{S_{i}} \frac{n_{i} C_{i}}{\prod_{\alpha_{i}}\left(p_{\alpha_{i}}^{2}-m_{\alpha_{i}}^{2}\right)},
$$

where $g$ is the gauge coupling constant, and $m_{\alpha_{i}}^{2}$ is the on-shell mass of the particle with momentum $p_{\alpha_{i}}$. The sum runs over the complete set $\Gamma$ of $m$-point $L$-loop graphs with only cubic vertices, including all possible permutations of external legs. The integrals are over the independent loop momenta $p_{l}$, and each graph is dressed with a kinematic numerator $n_{i}$ dependent upon the graph topology, a color factor $C_{i}$, and the propagator structure of the graph.

Contact terms are encoded by allowing the cubic graphs to be dressed with inverse propagators. Due to the ambiguity from assigning contact terms to different cubic graphs, there is some freedom in choosing the kinematic numerators $n_{i}$. A fortuitous representation is one where the kinematic numerator weights $n_{i}$ are color-dual $[1,2]$ — where they obey the same algebraic relations as the color (or flavor) weights $C_{i}$. At four points this is responsible for so-called radiation zeros $[59,60]$ and happens for all assignations to only cubic graphs at this multiplicity. It requires a specific generalized gauge choice at higher multiplicity ${ }^{3}$ and loop order, and can be made manifest in a wide web of theories [3], including nonsupersymmetric matter in the fundamental $[6-8,58]$. This means that for Jacobi-triple color weights, $C_{i}$, the kinematic numerators, $n_{i}$, satisfy the same anti-symmetry properties and Jacobi-like relations as the color factors. If the kinematic graph weights of a given amplitude satisfy the duality, we can replace the color factors in Equation (2.1) with another set of kinematic numerators

$$
\mathcal{M}_{m}^{(L)}=i^{L+1}\left(\frac{\kappa}{2}\right)^{m-2+2 L} \sum_{i \in \Gamma} \int \prod_{l=1}^{L} \frac{d^{D} p_{l}}{(2 \pi)^{D}} \frac{1}{S_{i}} \frac{n_{i} \tilde{n}_{i}}{\prod_{\alpha_{i}}\left(p_{\alpha_{i}}^{2}-m_{\alpha_{i}}^{2}\right)},
$$

where the tilde on $\tilde{n}$ signifies that there can be two distinct gauge theories. Any gaugeinvariance that was predicated on the algebraic properties of the color-weights are automatically inherited by the double-copy. Indeed, it is sufficient for only one of the numerator weights to make manifest the color kinematics duality. The expression obtained in Equation (2.2) is a gravitational amplitude. This generation of gravitational amplitudes is called double copy construction.

There are many possible combinations of kinematic numerators from different gauge theories, with varying degrees of supersymmetry, which lead to amplitudes in different gravitational theories - for a recent review of the relevant literature see, e.g. [3]. In this

\footnotetext{
${ }^{3}$ Generic assignations to cubic graphs will not respect Jacobi and antisymmetry, but the fact that there exists such a priveledged choice implies [1] a smaller basis $(n-3)$ ! for color-ordered Yang-Mills amplitudes at tree-level - evidently responsible for radiation zeroes at higher multiplicity as well [61].
} 
paper we will start with the double copy of massive scalar-QCD theory predictions given in ref. [8]. This generates predictions in a gravitational theory with propagating dilatons as well as antisymmetric tensor modes. In order to obtain amplitudes in Einstein-Hilbert gravity we will modify the method of maximal cuts to remove any non-gravitational propagating massless states. Additionally in the gravitational double-copy there can be novel local contact terms between massive matter. Such massive matter contact contributions are irrelevant to long-range predictions in any classical limit.

\subsection{Method of maximal cuts}

The method of maximal cuts iteratively constructs tree-level and the integrands of multiloop scattering amplitudes by inverting the sufficient verification condition of a loopintegrand satisfying all unitarity cuts. It is important to note that cut construction alone is only sufficient when there are no contact terms invisible to all factorization channels that one has not accounted for. Since unitarity cuts involving trees with uncut propagators span any unitarity cuts with additionally consistent cut propagators there is a natural hierarchy for construction. Namely one can build weights associated with graphs that first satisfy all possible cut conditions (all propagators put on shell), then consider what is required when cut conditions are relaxed to satisfy more inclusive cuts. Any cut-constructible missing information (encodable potentially in local Feynman rules) must be proportional to newly uncut propagators in order to have vanished when such newly-relevant propagators were on-shell. We specifically work here in (arbitrarily large) $D$-dimensions using formal polarizations and momentum invariants. This means that Lorentz products that might be related in three or four dimensions are allowed to be independent. This allows us to cut as many propagators simultaneously as we wish, whereas fixed dimensions introduce constraints ultimately imposed by fixed-dimension Gram determinants. Additionally this allows unitarity methods access to $D$-dimensional information important in dimensional regularization, that would otherwise be considered not "cut-constructable".

Consider a general unitarity cut with $k$ uncut propagators:

$$
\mathcal{C}^{N^{k} \mathrm{MC}}=\sum_{\text {states }} \mathcal{M}_{m(1)}^{\text {tree }} \ldots \mathcal{M}_{m(p)}^{\text {tree }} \quad k \equiv \sum_{i=1}^{p} m(i)-3,
$$

where $\mathcal{M}_{m(i)}^{\text {tree }}$ are tree-level $m(i)$ multiplicity gravity amplitudes, $k$ is the number of propagators that remain off-shell, and the sum runs over the possible states of the cut.

The first step in the method of maximal cuts is therefore to perform the maximal cuts of all relevant topologies of the amplitude, and compare with the sum over states of the product of trees described above. Thus for each maximal cut, which can be represented by a cubic graph (in arbitrarily large dimensions), all propagators are put on-shell. The tree amplitudes in a maximal cut are all three-point amplitudes, and so only one cubic graph contributes to each cut. The contributions of maximal cuts can be unambiguously assigned to those cubic topologies. For local representations there is no choice other than how to represent conservation of momentum zeros under cut conditions. The only potential missing contributions must be proportional to inverse-propagators (momentum zeros for 
all maximal cuts). To identify them we subsequently relax cut conditions. This subsequent step involves performing the next-to-maximal cuts (NMC) and assigning their contributions to any of the cubic graphs that contribute to that cut. For each next to maximal cut, only one of the cut conditions in a maximal cut is released, effectively returning propagators offshell, so for full (orderless) cuts - as relevant for gravitational or color-dressed theories at most $^{4}$ three graphs could contribute to each cut. For each such cut the only potentially new information will be proportional to the inverse of the newly uncut propagator, and this can be assigned or distributed amongst the three cubic topologies. One proceeds similarly, for the $\mathrm{N}^{2} \mathrm{MC}$ two propagators are held off-shell, and so on, until all potentially missing information is accounted for.

\section{Projective double-copy}

If one were to attempt to bootstrap pure Einstein-Hilbert gravitational amplitudes for four external gravitons from three-point amplitudes, using the method of maximal cuts alone, one runs into trouble immediately due to the four-point contact term required for linearized diffeomorphism invariance. Famously, gravitation requires additional contact terms at all multiplicity. At tree-level one can exploit analyticity properties to guarantee gauge-invariance via on-shell recursion $[62,63]$. Naive double-copy, which generalizes straightforwardly to loop-level, alleviates such worry, at the cost of potentially extra modes relative to pure-Einstein-Hilbert gravitation, and fixing a choice of theory dependent contact terms between massive-scalars. Any weight given to massive-scalar contacts are of course irrelevant in the classical limit. All supergravity theories, including the so-called $\mathcal{N}=0$ supergravity double-copy of pure Yang-Mills, share tree-level all-graviton amplitudes with pure Einstein-Hilbert gravitation. This of necessity changes at loop-level, where every state in the theory can contribute to loops. Famously, even at tree-level, this universality is also no longer the case when coupling to massive matter [58]. Critically, identifying these extra-mode contributions on any given amplitude is accessible through the method of maximal cuts.

We bootstrap pure-gravitational scattering between massive scalars via double-copy and the method of maximal cuts by only allowing gravitons to flow through the cuts involving massless propagators. To this end we use the $D_{s}$-dimensional physical state projector, described in [44] and references therein, to sew together the tree amplitudes,

$$
P^{\mu \nu \rho \sigma}(p, q)=\sum_{\text {pols. }} \varepsilon^{\mu \nu}(-p) \varepsilon^{\rho \sigma}(p)=\frac{1}{2}\left(P^{\mu \rho} P^{\nu \sigma}+P^{\mu \sigma} P^{\nu \rho}\right)-\frac{1}{D_{s}-2} P^{\mu \nu} P^{\rho \sigma},
$$

where

$$
P^{\mu \nu}=\eta^{\mu \nu}-\frac{q^{\mu} p^{\nu}+p^{\mu} q^{\nu}}{p \cdot q},
$$

where $q$ is an arbitrary null reference momenta that should factor out of any physical expression once the cut conditions and conservation of momenta have been imposed. In

\footnotetext{
${ }^{4}$ Flavor conservation can further restrict the number of potential graphs contributing to any cut.
} 
certain cases the state projector is equivalent to the simpler gauge-dependent projector,

$$
\sum_{\text {pols. }} \varepsilon_{\mu \nu}(-p) \varepsilon_{\rho \sigma}(p)=\frac{1}{2} \eta_{\mu \rho} \eta_{\nu \sigma}+\frac{1}{2} \eta_{\mu \sigma} \eta_{\nu \rho}-\frac{1}{D_{s}-2} \eta_{\mu \nu} \eta_{\rho \sigma}
$$

The state projector projects out non-gravitational states so that only gravitons are allowed to cross the cuts. We can then attribute any discrepancies between products of trees and the cut amplitude, or amplitude integrand, to excess states. We correct for these contributions by subtracting them from new higher-level contact terms as necessary. One can assign these contact contributions to cubic parent graphs in a manner that obeys the symmetries of those graphs (via ansatz and explicit symmetrization).

The initial contact contribution, involving non gravitationally mediated massless states, to each graph is given by that graph's maximal cut — where all propagators are cut:

$$
\Delta_{g}^{\mathrm{MC}}=\left.\mathcal{M}_{g}\right|^{\mathrm{MC}}-\sum_{\text {projected states }} \mathcal{M}_{3}^{\text {tree }} \cdots \mathcal{M}_{3}^{\text {tree }},
$$

where $\left.\mathcal{M}_{g}\right|^{\mathrm{MC}}$ is the maximal cut of graph $g$. The Einstein-Hilbert numerators, $n^{\mathrm{G}}$, that satisfy these cut conditions are given by subtracting off a topology-symmetrized $\tilde{\Delta}^{\mathrm{MC}}$ from the $\mathcal{N}=0$ numerator of the graph

$$
n_{g}^{\mathrm{G}}=n_{g}^{\mathcal{N}}-\tilde{\Delta}_{g}^{\mathrm{MC}}
$$

Any errors in $n^{\mathrm{G}}$ must be proportional to contact terms, but critically cannot involve contact terms due to all-graviton interaction, as those are correctly accounted for in the $\mathcal{N}=0$ supergravity numerators.

Higher cuts proceed as above, but the initial contribution is a $\Delta_{\text {contact }}^{\mathrm{N}^{k} \mathrm{MC}} \mid$ which must be assigned to cubic parent graph $n_{g}^{\mathrm{G}}$ via inverse propagators. For these higher point contact corrections we use $\tilde{\Delta}_{g}$ to represent the symmetrized corrections including the inverse propagators that target a particular graph $g$. The procedure terminates when all physical cut conditions are released.

\section{Extracting Einstein-Hilbert at tree and loop level}

Here we carry out the program of bootstrapping pure gravity amplitudes from double copies of massive scalars coupled to gluons, by projecting out excess states at the level of the numerator dressings. In [8] a set of color-dual kinematic numerators was determined for amplitudes with gluons and massive scalar particles in scalar QCD. The "naive double copy' numerators are simply squares of these

$$
n_{g}^{\mathcal{N}=0}=\left(n_{g}^{\mathrm{QCD}}\right)^{2}
$$

where $g$ is a cubic graph topology contributing to the amplitude, and $n_{g}^{\mathrm{QCD}}$ is the kinematic numerator dressing from the gauge theory amplitude. The pure gravity three-point graph 
numerators are simply given by the double copy of the corresponding kinematic dressings found in $[8]$

$$
\begin{aligned}
& n^{G}\left(\sum_{k_{2}}^{k_{3}} \begin{array}{l}
\vdots \\
k_{1}
\end{array}\right)=\left(k_{1} \cdot \varepsilon_{3}\right)^{2} \\
& n^{G}\left(\begin{array}{c}
k_{2} \\
\\
\sigma_{k_{1}} \\
\sigma^{\circ} \\
k_{k_{3}}
\end{array}\right)=\left(\left(k_{3} \cdot \varepsilon_{1}\right)\left(\varepsilon_{2} \cdot \varepsilon_{3}\right)-\left(k_{3} \cdot \varepsilon_{2}\right)\left(\varepsilon_{1} \cdot \varepsilon_{3}\right)+\left(k_{2} \cdot \varepsilon_{3}\right)\left(\varepsilon_{1} \cdot \varepsilon_{2}\right)\right)^{2} \text {. }
\end{aligned}
$$

We can now bootstrap from the above results to find the scattering amplitudes for massive scalars coupled gravitationally, including all appropriate gravitational self-interaction terms.

\subsection{Tree-level}

In addition to the graviton, the naive double-copy of the four-point tree-level amplitude with two massive scalars of distinct mass admits non-gravitational mediators, which we project out on cuts using the physical graviton state projector as described in section 3 . The tree-level case is relatively simple even at higher multiplicity, as only one channel is ever cut at a time. The entire procedure can be understood by simply considering the four-point example in detail. We tabulate all results with external massive scalars through six-point, including full amplitudes in the supplementary material.

The graph representation used here consists solely of cubic graphs, so the four-point amplitude involves a single graph topology,

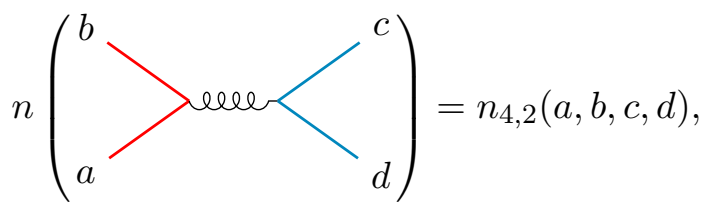

which is dressed with a functional kinematic numerator $n_{4,2}(a, b, c, d)$. The numerator function takes the ordered external legs $a, b, c, d$ as arguments, by which it is completely determined. The functional expression consists of Lorentz products of kinematic variables - such as momenta and polarization vectors - and must obey the same isomorphisms, or symmetries, as the corresponding graph topology. In [8] this kinematic numerator was determined for massive scalars coupled to gluons

$$
n_{4,2}^{\mathrm{QCD}}(a, b, c, d)=-\frac{1}{2}\left(k_{a} \cdot k_{b}+2 k_{b} \cdot k_{c}+m_{1}^{2}\right),
$$

where $m_{1}^{2}$ is the squared mass of the scalar $a, b$, and $k_{i} \cdot k_{j}$ is the Lorentz product of momenta. The $\mathcal{N}=0$ supergravity numerator is the square of the QCD numerator

$$
n_{4,2}^{\mathcal{N}=0}(a, b, c, d)=\left(n_{4,2}^{\mathrm{QCD}}(a, b, c, d)\right)^{2}=\frac{1}{4}\left(k_{a} \cdot k_{b}+2 k_{b} \cdot k_{c}+m_{1}^{2}\right)^{2} .
$$


For this amplitude the projective double-copy requires fixing the only factorization channel of the graph. We find the product of trees as described above using the state projector in equation (3.1),

$$
\begin{aligned}
\sum_{a}^{b} e_{s}^{c} & =\sum_{s} \mathcal{M}_{3}^{\text {trees }}\left(a, b, q^{s}\right) \mathcal{M}_{3}^{\text {trees }}\left(-q^{s}, c, d\right) \\
& =\left(k_{b} \cdot k_{d}\right)^{2}-\frac{m_{1}^{2} m_{2}^{2}}{\left(D_{s}-2\right)} .
\end{aligned}
$$

Recall, $D_{s}$ is the number of space-time dimensions arising from the trace of the metric, $\delta_{\mu}^{\mu}=D_{s}$, in the physical state projector. The cut amplitude is given by

$$
\left.\mathcal{M}_{4,2}^{\text {tree }}(a, b, c, d)\right|_{q^{2}=0} ^{\text {cut }}=\left.n_{4,2}^{\mathcal{N}=0}(a, b, c, d)\right|_{q^{2}=0} ^{\text {cut }}=\left(k_{b} \cdot k_{d}\right)^{2} .
$$

The extra-state difference between the factorized numerators is identified,

$$
\Delta_{4,2}=\left.\mathcal{M}_{4,2}^{\text {tree }}(a, b, c, d)\right|_{q^{2}=0} ^{\text {cut }}-\sum_{s} \mathcal{M}_{3}^{\text {trees }}\left(a^{m_{1}}, b^{m_{1}}, q^{s}\right) \mathcal{M}_{3}^{\text {trees }}\left(-q^{\bar{s}}, c^{m_{2}}, d^{m_{2}}\right)=\frac{m_{1}^{2} m_{2}^{2}}{\left(D_{s}-2\right)},
$$

where massive particles are denoted in the tree-labels by a superscript $m_{i}$, and the two $\mathcal{M}_{3}^{\text {tree }}$ are the 3 -point gravity tree amplitudes given in equation (4.2). We then proceed to update the numerator function for pure-gravity exchange by subtracting the extra-state dimension

$$
n_{4,2}^{G}(a, b, c, d)=n_{4,2}^{\mathcal{N}=0}(a, b, c, d)-\Delta_{4,2}=\frac{1}{4}\left(k_{a} \cdot k_{b}+2 k_{b} \cdot k_{c}+m_{1}^{2}\right)^{2}-\frac{m_{1}^{2} m_{2}^{2}}{\left(D_{s}-2\right)} .
$$

The full amplitude for two-to-two scattering at tree level in Einstein-Hilbert gravity (cf. ref. [58]) is then found to be,

$$
\mathcal{M}_{4}^{\text {tree }}\left(a^{m_{1}}, b^{m_{1}}, c^{m_{2}}, d^{m_{2}}\right)=\frac{\frac{1}{4}\left(k_{a} \cdot k_{b}+2 k_{b} \cdot k_{c}+m_{1}^{2}\right)^{2}-\frac{m_{1}^{2} m_{2}^{2}}{\left(D_{s}-2\right)}}{(a+b)^{2}} .
$$

Higher multiplicity proceeds as above where first one considers any corrections from maximal cuts and proceeds to release all cut conditions. The $\mathcal{N}=0$ supergravity dressings and excess state contributions $\Delta$ are shown in table 1 for amplitudes with one massive scalar. As seen from the table there are no excess state corrections for the tree amplitudes with a single scalar line. This is not unexpected, as the states are expected to propagate between scalar lines.

The tree-level dressings for four-point and five-point amplitudes with two scalar lines are shown in table 2, and the corrections for the six-point amplitude with two massive scalars are shown in table 3 . These graphs do require corrections from $\mathcal{N}=0 \mathrm{SG}$. We can now easily calculate the first correction to massive scalar scattering with radiation. 


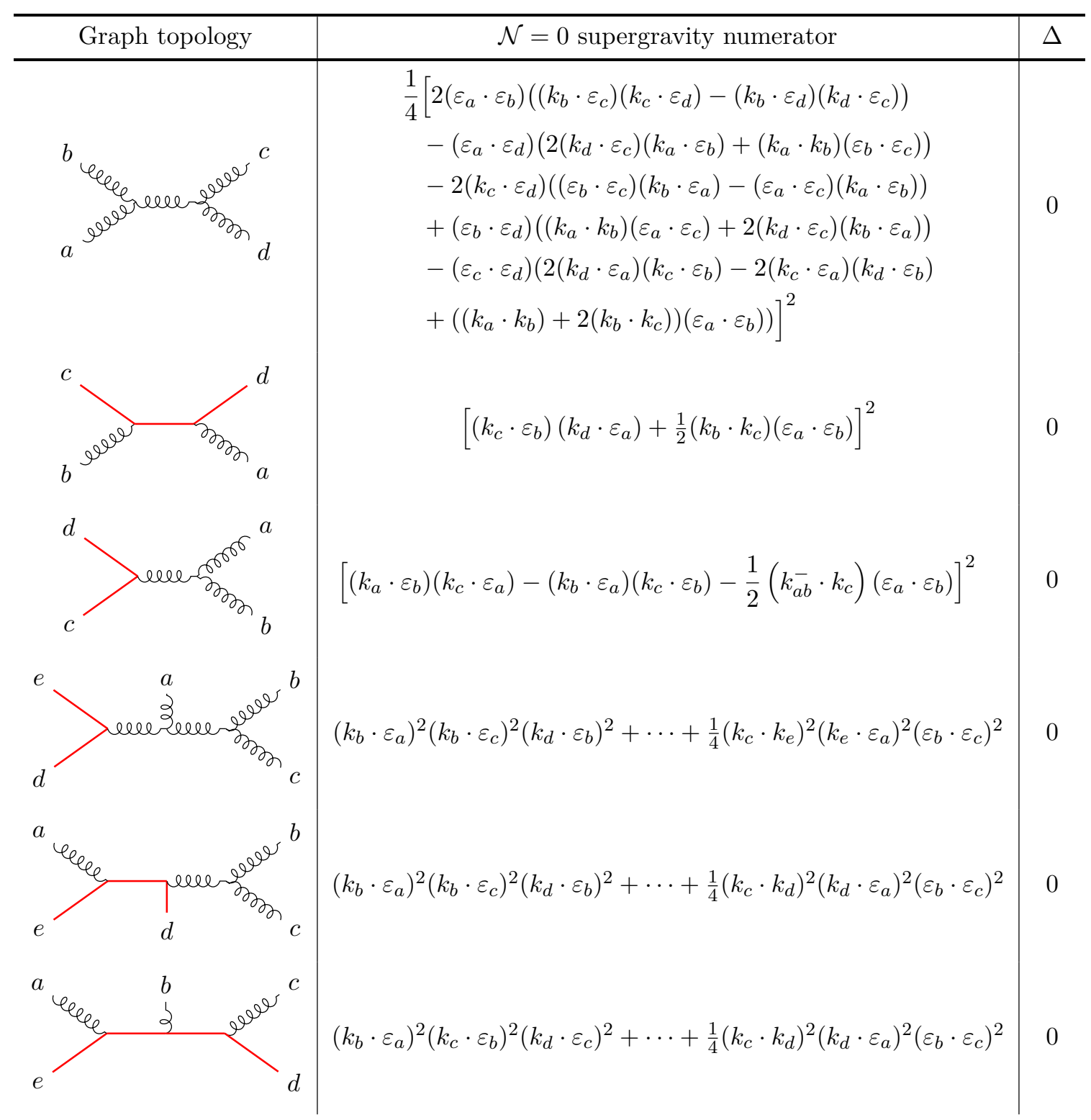

Table 1. Tree amplitude graphs for one massive scalar at four and five-point, where $k_{i j}=k_{i}+k_{j}$ and $k_{i j}^{-}=k_{i}-k_{j}$. The correction $\Delta$ should be subtracted from the $\mathcal{N}=0$ supergravity dressing to obtain the pure graviton numerator.

The amplitude gets contributions from the second topology in table 2 and four differentlylabeled versions of the third topology in table 2 , using $k_{i j}^{-} \equiv k_{i}-k_{j}$,

$$
\begin{aligned}
& \mathcal{M}_{5}^{\text {tree }}\left(k_{1}, k_{2}^{m_{1}}, k_{3}^{m_{1}}, k_{4}^{m_{2}}, k_{5}^{m_{2}}\right)= \\
& \frac{\left(2\left(k_{3} \cdot k_{45}^{-}\right)\left(k_{2} \cdot \varepsilon_{1}\right)+\left(k_{1} \cdot k_{2}\right)\left(k_{45}^{-} \cdot \varepsilon_{1}\right)\right)^{2}-\frac{1}{D_{s}-2} 16 m_{1}^{2} m_{2}^{2}\left(k_{2} \cdot \varepsilon_{1}\right)^{2}}{16\left(k_{4}+k_{5}\right)^{2}\left(\left(k_{1}+k_{2}\right)^{2}-m_{1}^{2}\right)} \\
& +\frac{\left(2\left(k_{2} \cdot k_{45}^{-}\right)\left(k_{3} \cdot \varepsilon_{1}\right)+\left(k_{1} \cdot k_{3}\right)\left(k_{45}^{-} \cdot \varepsilon_{1}\right)\right)^{2}-\frac{1}{D_{s}-2} 16 m_{1}^{2} m_{2}^{2}\left(k_{3} \cdot \varepsilon_{1}\right)^{2}}{16\left(k_{4}+k_{5}\right)^{2}\left(\left(k_{1}+k_{3}\right)^{2}-m_{1}^{2}\right)}
\end{aligned}
$$




\begin{tabular}{|c|c|c|}
\hline Graph topology & $\mathcal{N}=0$ supergravity numerator & $\Delta$ \\
\hline & $\frac{1}{4}\left(k_{a} \cdot k_{b}+2 k_{b} \cdot k_{c}+m_{1}^{2}\right)^{2}$ & $\frac{m_{1}^{2} m_{2}^{2}}{D_{s}-2}$ \\
\hline & $\begin{aligned} \frac{1}{16}\left[\left(k_{b c}^{-} \cdot k_{1}\right)\left(k_{d e}^{-} \cdot \varepsilon_{1}\right)\right. & +2\left(k_{c} \cdot k_{d e}^{-}\right)\left(k_{b} \cdot \varepsilon_{a}\right) \\
& \left.-2\left(k_{b} \cdot k_{d e}^{-}\right)\left(k_{c} \cdot \varepsilon_{a}\right)\right]^{2}\end{aligned}$ & $\frac{m_{1}^{2} m_{2}^{2}}{D_{s}-2}\left(k_{d e} \cdot \varepsilon_{a}\right)^{2}$ \\
\hline & $\frac{1}{16}\left[\left(k_{c e} \cdot k_{d e}\right)\left(k_{d e}^{-} \cdot \varepsilon_{a}\right)+2\left(k_{c} \cdot k_{d e}^{-}\right)\left(k_{b} \cdot \varepsilon_{a}\right)\right]^{2}$ & $\frac{m_{1}^{2} m_{2}^{2}}{D_{s}-2}\left(k_{b} \cdot \varepsilon_{a}\right)^{2}$ \\
\hline
\end{tabular}

Table 2. Tree amplitude graphs for two massive scalars, at four and five-point, where $k_{i j}=k_{i}+k_{j}$ and $k_{i j}^{-}=k_{i}-k_{j}$. The correction $\Delta$ should be subtracted from the $\mathcal{N}=0$ supergravity dressing to obtain the pure graviton numerator.

$$
\begin{aligned}
& +\frac{\left(2\left(k_{4} \cdot k_{23}^{-}\right)\left(k_{5} \cdot \varepsilon_{1}\right)+\left(k_{1} \cdot k_{5}\right)\left(k_{23}^{-} \cdot \varepsilon_{1}\right)\right)^{2}-\frac{1}{D_{s}-2} 16 m_{1}^{2} m_{2}^{2}\left(k_{5} \cdot \varepsilon_{1}\right)^{2}}{16\left(k_{2}+k_{3}\right)^{2}\left(\left(k_{1}+k_{5}\right)^{2}-m_{2}^{2}\right)} \\
& +\frac{\left(2\left(k_{5} \cdot k_{23}^{-}\right)\left(k_{4} \cdot \varepsilon_{1}\right)+\left(k_{1} \cdot k_{4}\right)\left(k_{23}^{-} \cdot \varepsilon_{1}\right)\right)^{2}-\frac{1}{D_{s}-2} 16 m_{1}^{2} m_{2}^{2}\left(k_{4} \cdot \varepsilon_{1}\right)^{2}}{16\left(k_{2}+k_{3}\right)^{2}\left(\left(k_{1}+k_{4}\right)^{2}-m_{2}^{2}\right)} \\
& +\frac{\left(\left(k_{23}^{-} \cdot k_{1}\right)\left(k_{45}^{-} \cdot \varepsilon_{1}\right)+2\left(k_{3} \cdot k_{45}^{-}\right)\left(k_{2} \cdot \varepsilon_{1}\right)-2\left(k_{2} \cdot k_{45}^{-}\right)\left(k_{3} \cdot \varepsilon_{1}\right)\right)^{2}-\frac{1}{D_{s}-2} 16 m_{1}^{2} m_{2}^{2}\left(k_{45} \cdot \varepsilon_{1}\right)^{2}}{16\left(k_{2}+k_{3}\right)^{2}\left(k_{4}+k_{5}\right)^{2}} .
\end{aligned}
$$

\subsection{Loop level}

The physical cuts relevant to one-loop are relatively straightforward. For both four-point one-loop and five-point one-loop reduction to standard integral basis means satisfying cuts to tree-level under the following restrictions:

- Every bubble cut (two-particle cut) involves physical tree amplitudes (multiplicity four or higher). These are $\mathrm{N}^{2} \mathrm{MC} 1-\mathrm{N}^{2} \mathrm{MC} 4$ in figure 4 for the four-point amplitude, and $\mathrm{N}^{3} \mathrm{MC} 1-\mathrm{N}^{3} \mathrm{MC} 5$ in figure 11 for the five-point amplitude.

- Every triangle cut (three-particle cut) must involve the loop momenta flowing through each tree. These are cuts NMC1, NMC2 and NMC3 in figure 2 for the four-point amplitude, and $\mathrm{N}^{2} \mathrm{MC} 1-\mathrm{N}^{2} \mathrm{MC} 11$ in figure 9.

- Every box cut (four-particle cut) for the five-point amplitude, must involve the loopmomenta flowing through each tree. These are cut MC1 in figure 1 for the four-point amplitude and cuts NMC1-NMC7 in figure 7. 


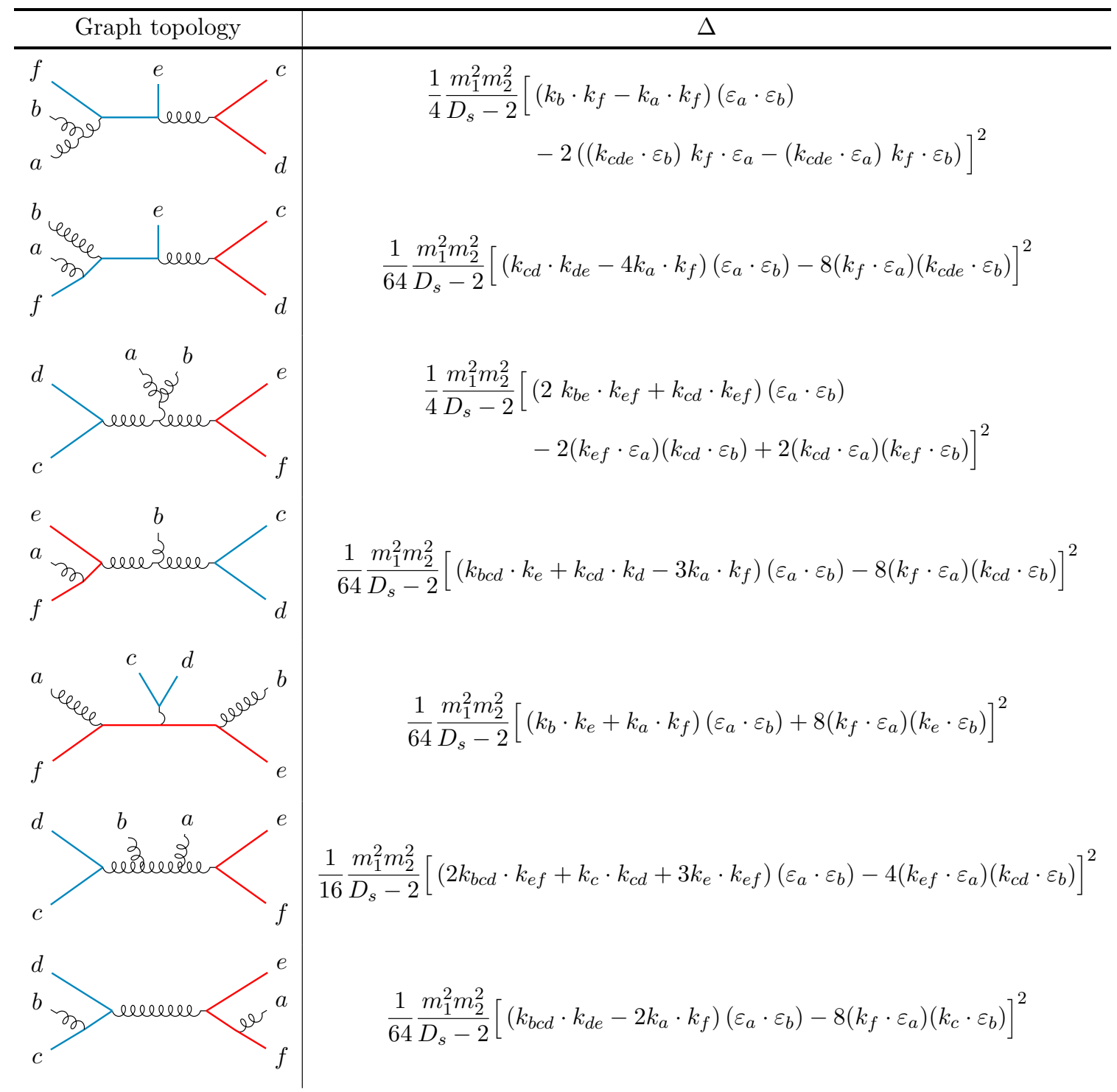

Table 3. The graph topologies for the six-point tree amplitude for two distinct-mass scalars, and the corresponding contributions from non-gravitational states $\Delta$, where $k_{i j}=k_{i}+k_{j}$.

This set of restrictions precludes one-loop snail ${ }^{5}$ diagrams as well as tadpole diagrams from contributing to the cuts. Any such contributions are not cut constructible with standard (non-regulated) approaches - even in arbitrary dimensions - representing a distinct set of considerations, but one absolutely complementary and compatible with unitary methods as per ref. [65]. As such for each multiplicity at one loop, we only concern ourselves with the series of maximal cuts which can contribute to these physical cuts. This entirely spans any information relevant to the classical $\hbar \rightarrow 0$ limit.

\footnotetext{
${ }^{5}$ Apparently one-particle reducible topologies can contribute in an all cubic diagram basis, but only when dressed with appropriate inverse-propagators to actually be encoding one-particle irreducible contactdiagrams [64].
} 


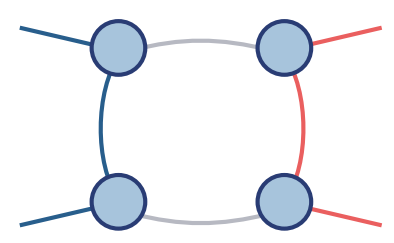

(a) MC1.

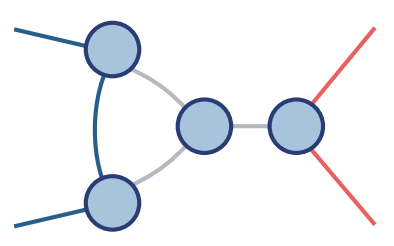

(b) $\mathrm{MC} 2$.

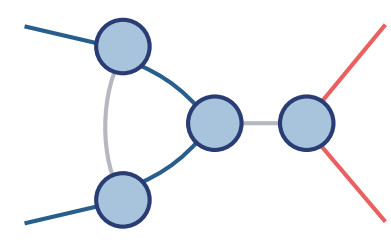

(c) MC3.

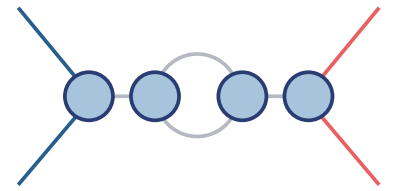

(d) MC4.

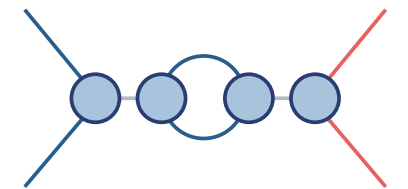

(e) MC5.

Figure 1. All distinct maximal cuts of the one-loop four-point amplitude, not including tadpole graphs. Blue blobs represent three point amplitudes, and exposed legs represent on-shell propagators.

\subsubsection{Four-point one-loop}

Projective double copy allows us to construct the Einstein-Hilbert gravity numerator dressings for the graph topologies of the one-loop four-point amplitude that could possibly be relevant in the classical limit. The maximal cuts expose the information that is inherent to each graph topology, i.e. terms that cannot be moved to other cubic graphs. The next to maximal cuts, or $\mathrm{N}$-maximal cuts, in turn provide contact terms that can be distributed amongst the uncut propagators of the given cut. At this point a representation choice can thus be made as to where to assign these contributions. Some N-maximal cuts are delicate. Delicate next-to-maximal cuts have to deal with infinite poles that arrise after one of the maximal cut conditions is released exposing a vanishing propagator. This can occur when conservation of momentum forces an uncut propagator to be equivalent to a propagator with on-shell conditions imposed. The information that is missed by omitting these cuts can, however, be retrieved at the level of next-to-next-to maximal cuts, or $\mathrm{N}^{2}$-maximal cuts. This type of information shows up as a propagator with some kinematic coefficient, and must therefore be attributed to the corresponding graph topology. We find that the Einstein-Hilbert numerators are corrected by up to $\mathrm{N}^{2}$-maximal cuts relative to the $\mathcal{N}=0$ supergravity kinematic weights.

The first step is to calculate all maximal cuts. At one-loop the four-point amplitude has five cubic graph topologies which can ever contribute to the physical cuts, ${ }^{6}$ whose topologies can label potential maximal cuts. We present these in figure 1.

For the first consideration of physically relevant information, we now provide details for calculation of the cut MC1 (cf. figure 1) as an example. All propagators are on-shell and so the left-hand side of Equation (2.3) is simply the numerator of the box

$$
\left.\mathcal{M}_{4}^{1 \text {-loop }}\right|_{\mathrm{MC} 1} ^{\text {cut }}=\left.n_{\mathcal{N}=0}\left(\begin{array}{l}
2 \\
1
\end{array}\right]\left[\begin{array}{l}
3 \\
4
\end{array}\right)\right|_{\mathrm{MC} 1} ^{\text {cut }}=\left(k_{1} \cdot k_{4}\right)^{4},
$$

\footnotetext{
${ }^{6}$ We do not include here contacts associated with cubic graphs like bubbles on external legs nor massive tadpole graphs.
} 
where $\ell$ is the loop momenta and we have imposed the cut conditions $\ell^{2}=0,\left(\ell-k_{1}\right)^{2}=m_{1}^{2}$, $\left(\ell-k_{1}-k_{2}\right)^{2}=0$ and $\left(\ell+k_{4}\right)^{2}=m_{2}^{2}$. The right-hand side of Equation (2.3) is the state sum over the product of three-point tree amplitudes,

$$
\begin{aligned}
\left.\mathcal{M}^{\text {trees }}\right|_{\mathrm{MC} 1}= & \sum_{\text {states }} \mathcal{M}_{3}^{\text {tree }}\left(k_{1}^{m_{1}}, l_{1}^{m_{1}}, l_{3}^{s_{3}}\right) \mathcal{M}_{3}^{\text {tree }}\left(-l_{1}^{m_{1}}, k_{2}^{m_{1}}, l_{2}^{s_{2}}\right) \\
& \times \mathcal{M}_{3}^{\text {tree }}\left(-l_{2}^{\bar{s}_{2}}, k_{3}^{m_{2}},-l_{4}^{m_{2}}\right) \mathcal{M}_{3}^{\text {tree }}\left(l_{4}^{m_{2}}, k_{4}^{m_{2}},-l_{3}^{\bar{s}_{3}}\right) \\
= & \sum_{\text {states }}\left(l_{1} \cdot \varepsilon\left(l_{3}\right)\right)^{2}\left(l_{1} \cdot \varepsilon\left(l_{2}\right)\right)^{2}\left(l_{4} \cdot \varepsilon\left(-l_{2}\right)\right)^{2}\left(l_{4} \cdot \varepsilon\left(-l_{3}\right)\right)^{2} .
\end{aligned}
$$

Note that $\varepsilon\left(l_{i}\right) \neq \varepsilon\left(-l_{i}\right)$. Using the state projector to sum over polarizations, we find

$$
\left.\mathcal{M}^{\text {trees }}\right|_{\mathrm{MC} 1}=\left(\frac{m_{1}^{2} m_{2}^{2}}{D_{s}-2}-\left(k_{1} \cdot k_{4}\right)^{2}\right)^{2} .
$$

Similarly to tree-level, the contribution from non-gravitational states is given by the difference between Equation (4.13) and Equation (4.15)

$$
\begin{aligned}
\Delta^{\mathrm{MC} 1} & =\left.\mathcal{M}_{4}^{1 \text {-loop }}\right|_{\mathrm{MC} 1} ^{\mathrm{cut}}-\left.\mathcal{M}^{\text {trees }}\right|_{\mathrm{MC} 1} \\
& =2 m_{1}^{2} m_{2}^{2} \frac{\left(k_{1} \cdot k_{4}\right)^{2}}{D_{s}-2}-\frac{\left(m_{1}^{2} m_{2}^{2}\right)^{2}}{\left(D_{s}-2\right)^{2}}
\end{aligned}
$$

As mentioned, the functional numerators must obey all the isomorphisms of the graph topologies they describe - such as $k_{1} \leftrightarrow k_{2}, k_{3} \leftrightarrow k_{4}$ for the box graph - and $\Delta^{\mathrm{MC1}}$ is symmetrized accordingly. In this particular case the expression already obeys the relevant isomorphisms, and we have

$$
\hat{\Delta}^{\mathrm{MC} 1}=-\frac{\left(m_{1}^{2} m_{2}^{2}\right)^{2}}{\left(D_{s}-2\right)^{2}}+\frac{2 m_{1}^{2} m_{2}^{2}}{D_{s}-2}\left(k_{1} \cdot k_{4}\right)^{2} .
$$

The constructed Einstein-Hilbert numerator is then given by

$$
n_{G}^{\text {box }}=n_{\mathcal{N}=0}^{\text {box }}-\hat{\Delta}^{\mathrm{MC} 1}
$$

The $\Delta$ contributions before symmetrization from the maximal cuts of the one-loop amplitude can be found in table 4 .

Updating numerators on the $\mathrm{N}^{k}$-maximal cuts is a similar procedure, and the nextto-maximal cuts (NMC) can be found in figure 2. In the figure, the light blue blobs represent three-point tree amplitudes, and darker blobs represent higher-point trees where cut conditions have been released. The maximal cuts fix all the information inherent to each graph, and excess state contributions from higher-level cuts are therefore contact terms. As we have chosen to represent the amplitudes exclusively in terms of cubic graphs, these terms are associated with uncut propagators of the cut graph topologies. For example, 


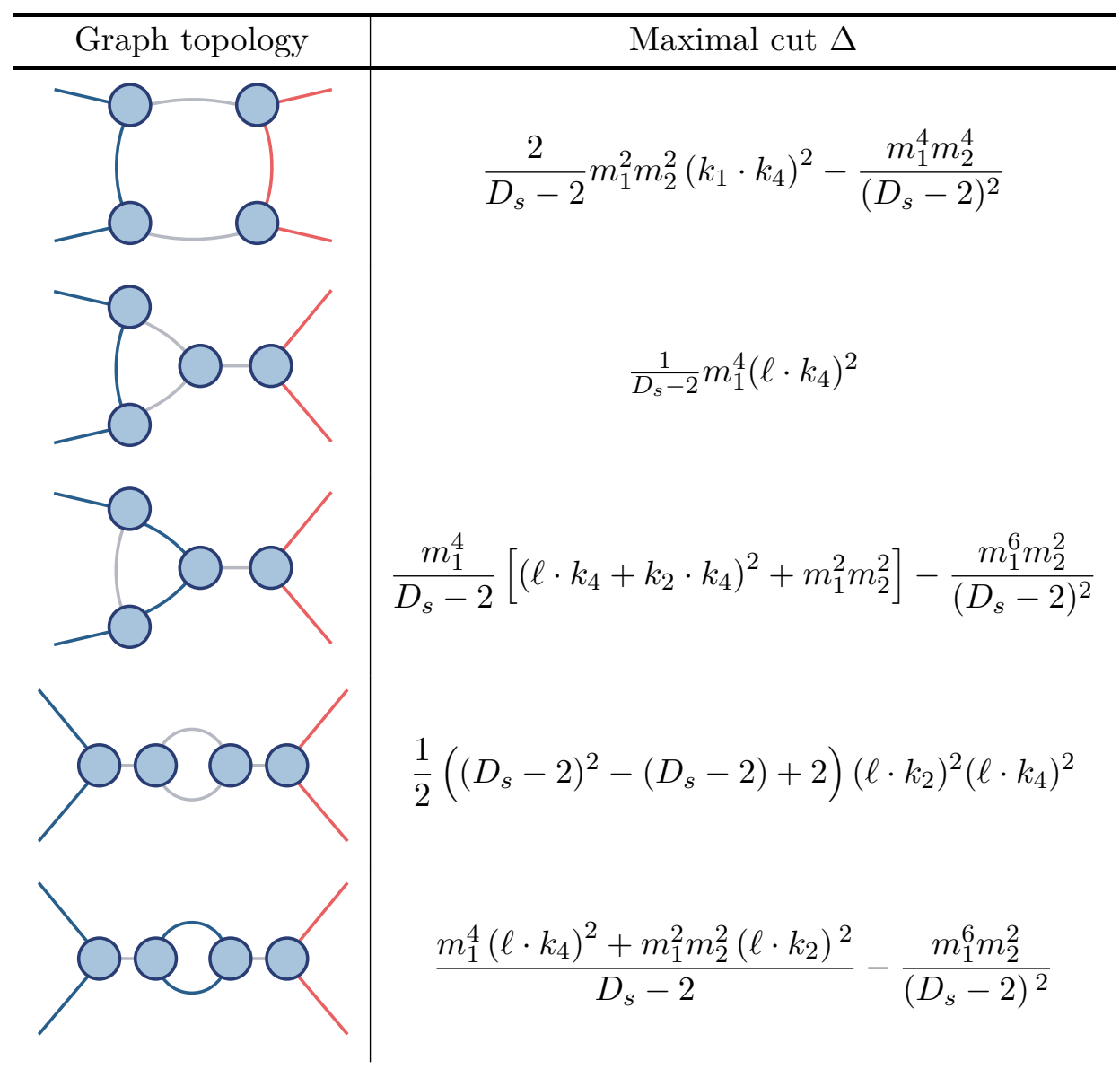

Table 4. The $\Delta$ contributions from the maximal cuts of the one-loop four-point amplitude, not including tadpole graphs. Blue blobs represent three point amplitudes, and exposed legs represent on-shell propagators.

consider the next-to-maximal cut NMC1, which yields the following additional information

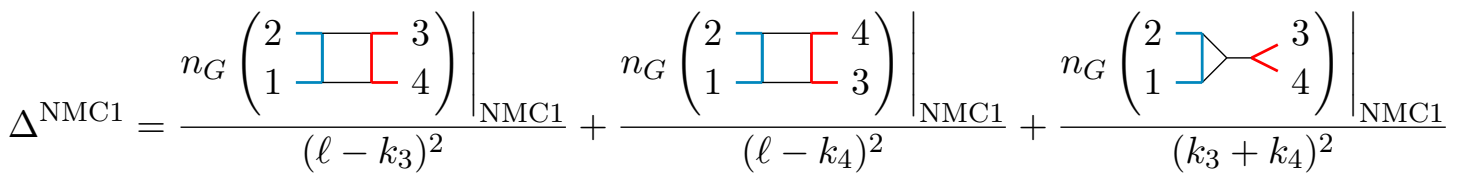

$$
\begin{aligned}
& -\sum_{\text {states }} \mathcal{M}_{3}^{\text {tree }}\left(k_{1}^{m_{1}}, l_{1}^{m_{1}}, l_{3}^{s_{3}}\right) \mathcal{M}_{3}^{\text {tree }}\left(-l_{1}^{m_{1}}, k_{2}^{m_{1}}, l_{2}^{s_{2}}\right) \mathcal{M}_{4}^{\text {tree }}\left(-l_{2}^{\bar{s}_{2}},-l_{3}^{\bar{s}_{3}}, k_{3}^{m_{2}}, k_{4}^{m_{2}}\right) \\
& =-\frac{m_{1}^{2}\left(m_{1}^{2}-4 m_{2}^{2}\right)}{8\left(D_{s}-2\right)}\left(k_{3}+k_{4}\right)^{2}-\frac{m_{1}^{4} m_{2}^{2}}{\left(D_{s}-2\right)^{2}},
\end{aligned}
$$

where $k_{i j}=k_{i}+k_{j}$. The resulting expression is a contact term which we are free to attribute to an uncut propagator in the box or triangle graph. We choose the triangle graph, whose uncut propagator is $\left(k_{3}+k_{4}\right)^{2}$. The correction to the Einstein-Hilbert numerator of the $\mathrm{MC} 2$ topology is then given by

$$
\hat{\Delta}_{\mathrm{NMC}}=-\left(k_{3}+k_{4}\right)^{2}\left[\frac{m_{1}^{2}\left(m_{1}^{2}-4 m_{2}^{2}\right)\left(k_{3}+k_{4}\right)^{2}}{8\left(D_{s}-2\right)}+\frac{m_{2}^{2} m_{1}^{4}}{\left(D_{s}-2\right)^{2}}\right],
$$




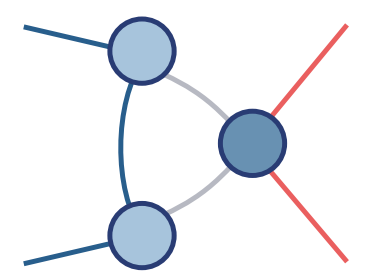

(a) NMC1.

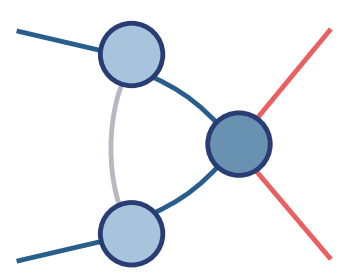

(b) NMC2.

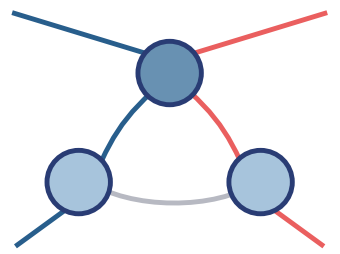

(c) NMC3.

Figure 2. Next-to-maximal cuts of the one-loop four-point amplitude. Light blue blobs represent three-point amplitudes, dark blobs represent four-point amplitudes, and exposed legs represent onshell propagators.

$-\left(k_{3}+k_{4}\right)^{2} \times\left[\frac{m_{1}^{2}\left(m_{1}^{2}-4 m_{2}^{2}\right)\left(k_{3}+k_{4}\right)^{2}}{8\left(D_{s}-2\right)}+\frac{m_{1}^{4} m_{2}^{2}}{\left(D_{s}-2\right)^{2}}\right]$
Graph topology
$\left(k_{1}+k_{2}+\ell\right)^{2} \times\left[m_{1}^{2} m_{2}^{2} \frac{\left(\ell \cdot k_{1}-\ell \cdot k_{4}\right)+2\left(3 k_{1} \cdot k_{4}-k_{2} \cdot k_{4}\right)}{16\left(D_{s}-2\right)}\right]$

Table 5. The symmetrized $\hat{\Delta}$ contributions from the well-defined next-to-maximal cuts of the one-loop four-point amplitude attributed to inverse propagators. Light blue blobs represent threepoint amplitudes, darker blobs represent four-point amplitudes and exposed legs represent on-shell propagators.

where we have highlighted the propagator prefactor of the expression, which is kept explicit to ensure the vanishing of this term in the maximal cut MC2.

Here one can choose representations that satisfy either ${ }^{7}$ adjoint-type or fundamentaltype scalar single-copy origins. For expedience in this paper and supplementary material we collapse to adjoint-type scalars as the expressions are relatively compact. The remaining next-to-maximal cuts in figure 2 give similar corrections to the gravity numerators, and are shown in table 5 with the corresponding $\hat{\Delta}_{\mathrm{NMC}}$ terms and the topology to which the new term is attributed.

Some next-to-maximal cuts are delicate. Cuts involving one collapsed propagator of bubble graphs such as NMC4-6 in figure 3, have infinite poles that blow up as cut conditions

\footnotetext{
${ }^{7}$ Or both.
} 


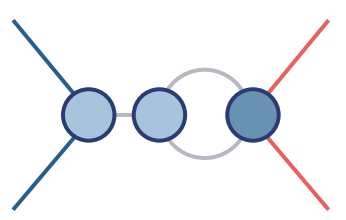

(a) NMC4.

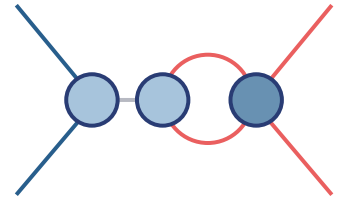

(b) NMC5.

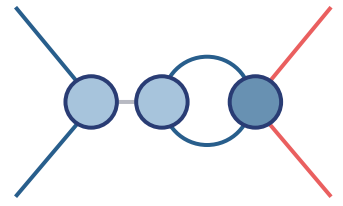

(c) NMC6.

Figure 3. Delicate next-to-maximal cuts of the one-loop four-point amplitude excluding snail and tadpole cuts. Light blue blobs represent three point amplitudes, darker blobs represent four-point amplitudes, and exposed legs represent on-shell propagators.

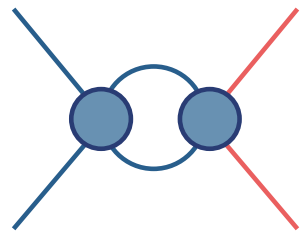

(a) $\mathrm{N}^{2} \mathrm{MC} 1$.

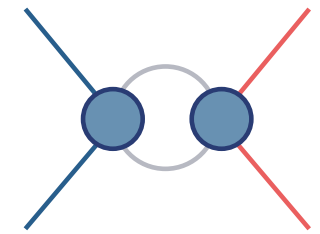

(b) $\mathrm{N}^{2} \mathrm{MC} 2$.

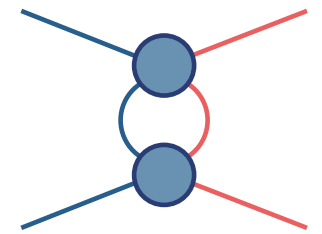

(c) $\mathrm{N}^{2} \mathrm{MC} 3$.

Figure 4. All next-to-next-to-maximal cuts of the one-loop four-point amplitude, not including tadpole graphs. Dark blue blobs represent four-point amplitudes, and exposed legs represent onshell propagators.

are imposed on the fully dressed graphs. By construction the numerator dressings are made up of a minimal kinematic basis where conservation of momentum has been imposed. This means that inverse propagators in the numerators - which should cancel against propagators in the denominator - are rewritten in a form where these cancellations do not occur. The cuts, as written, therefore naively go to infinity as propagators in the denominator are sent to zero. There is an important point associated with cut construction - one need not consider a cut if one can access all its information by considering a cut that spans it. Typically verification of an integrand will consider a very small number of cuts - a minimal spanning set. Because of the number of graphs (often the same topology but relabeled) can contribute to such minimal cuts, we make our lives easier starting with cuts that admit the fewest graphs rather than the most. However we do not need to let infinities get in our way, nor worry about delicate regularization, we can simply consider the set of well-behaved cuts that span these cuts and extract the information we need from those cuts. Any potentially physically relevant information contained in the delicate cuts can instead be extracted using the $\mathrm{N}^{2}$-maximal cuts (cf. figure 4).

Consider for example the $\mathrm{N}^{2}$-maximal cut $\mathrm{N}^{2} \mathrm{MC} 1$ in figure 4. This cut is equivalent to NMC6 with an additional propagator, $\left(k_{1}+k_{2}\right)^{2}$, put off-shell. The excess state term $\Delta$ for this cut is

$$
\Delta^{\mathrm{N}^{2} \mathrm{MC} 1}=-\frac{m_{1}^{2}}{16\left(D_{s}-2\right)}\left[\frac{12 m_{1}^{2} m_{2}^{2}}{\left(k_{3}+k_{4}\right)^{2}}-6 m_{2}^{2}+m_{1}^{2}\right],
$$

where we see that this is not a pure contact term. We see the contribution of a manifest propagator, so in this $\mathrm{N}^{2} \mathrm{MC}$ we have found the excess state contribution to one of the 
propagators in a previously skipped NMC cut. We attribute this information to the topology MC5 (massive bubble), by multiplying by appropriate combinations of the two uncut propagators $\left(k_{1}+k_{2}\right)^{2}=\left(k_{3}+k_{4}\right)^{2}$ which yields two distinct contact terms:

$$
\begin{aligned}
\hat{\Delta}^{\mathrm{N}^{2} \mathrm{MC} 1}= & \left(k_{3}+k_{4}\right)^{2} \times \frac{-3 m_{1}^{4} m_{2}^{2}}{4\left(D_{s}-2\right)} \\
& +\left(k_{1}+k_{2}\right)^{2}\left(k_{3}+k_{4}\right)^{2} \times \frac{\left(6 m_{1}^{2} m_{2}^{2}-m_{1}^{4}\right)}{16\left(D_{s}-2\right)} .
\end{aligned}
$$

As the first is proportional to a single uncut propagator, we see that it contains information relevant to a cut like NMC6. Of course because of conservation of momentum it vanishes when $\left(k_{1}+k_{2}\right)^{2}$ is on-shell, which is why it was sensible to wait until this $\mathrm{N}^{2}$-maximal cut to figure out where it belongs. The second term is proportional to the two propagators whose cut conditions are released at $\mathrm{N}^{2}$-maximal level, and so is inherently a $\mathrm{N}^{2} \mathrm{MC}$ correction.

Of the $\mathrm{N}^{2}$-maximal cuts shown in figure 4 the cuts $\mathrm{N}^{2} \mathrm{MC} 1$ and $\mathrm{N}^{2} \mathrm{MC} 2$ yield numerator corrections, and the remaining cut can be used for verification. The symmetrized corrections for the $\mathrm{N}^{2} \mathrm{MC}$ are shown in table 6 , with the propagator prefactors that correspond to attributing the cut information to the topologies shown in the right column.

All five maximal cuts of the four-point one-loop amplitude, not including snail or tadpole graphs, are shown in figure 1. All the cuts in the figure give corrections which are inherent to the gravity numerators of these topologies. The three next-to-maximal cuts shown in figure 2 are well-defined, and removing cut conditions do not result in infinite poles. The first of these cuts, NMC1, exposes a correction that can be attributed to one of two graph topologies (corresponding to MC1 and MC2 in figure 1). The remaining two well-defined cuts - NMC2, NMC3 - also give corrections, but they involve four-point tree amplitudes with two massive scalars, which means only one topology contributes to each of these cuts. In figure 3 we show three delicate next to maximal cuts, whose relevant information can be more easily extracted by further relaxing cut conditions. Finally, the $\mathrm{N}^{2}$-maximal cuts are shown in figure 4. The two first cuts $-\mathrm{N}^{2} \mathrm{MC} 1$ and $\mathrm{N}^{2} \mathrm{MC} 2-$ give corrections to the numerators, including information from the skipped N-maximal cuts. The third cut, $\mathrm{N}^{2} \mathrm{MC} 3$, receives no correction.

\subsubsection{Five-point one-loop}

We proceed with the projective double copy to find the one-loop gravitational radiative correction to the two-to-two scattering of massive scalars. The procedure at five-points is the same as demonstrated at four-points, again starting with the maximal cuts. The 24 maximal cuts relevant to the physical cuts for this five-point amplitude are shown in figures 5-6, excluding both tadpole and snail topologies. All the maximal cuts give excess state corrections to the corresponding graph numerators.

The 30 next-to-maximal cuts can be found in figures 7-8, and all of these cuts give corrections to the numerators. There are $19 \mathrm{~N}^{2}$-maximal cuts which are shown in figures 9 10. In contrast to the next-to-maximal cuts, not all of these yield corrections. The cuts $\mathrm{N}^{2} \mathrm{MC} 1, \mathrm{~N}^{2} \mathrm{MC} 3, \mathrm{~N}^{2} \mathrm{MC} 11$ and $\mathrm{N}^{2} \mathrm{MC} 19$ give no contributions, while the remaining 15 cuts do correct for excess states. At five-point one-loop the highest order of corrections come 


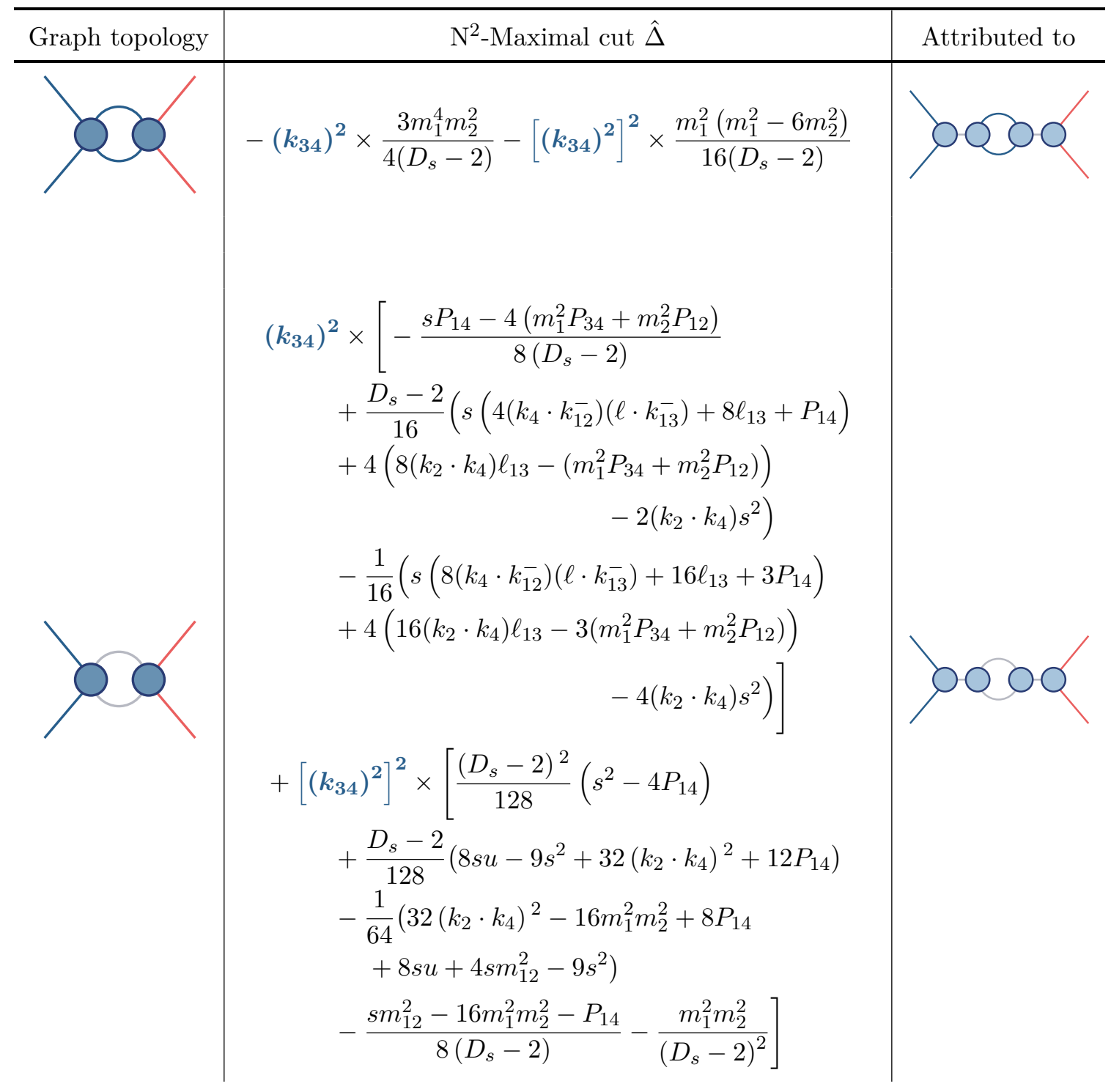

Table 6. The symmetrized $\hat{\Delta}$ contributions from the well-defined $\mathrm{N}^{2}$-maximal cuts of the oneloop four-point amplitude attributed to inverse propagators. Light blue blobs represent threepoint amplitudes, darker blobs represent four-point amplitudes, and exposed legs represent onshell propagators. Here $\ell_{13}=\left(\ell \cdot k_{1}\right)\left(\ell \cdot k_{3}\right), m_{12}^{2} \equiv m_{1}^{2}+m_{2}^{2}, s \equiv\left(k_{3}+k_{4}\right)^{2}, u \equiv\left(k_{2}+k_{4}\right)^{2}$, $k_{i j}=k_{i}+k_{j}, k_{i j}^{-}=k_{i}-k_{j}, P_{12}=\left(\ell \cdot k_{1}\right)^{2}+\left(\ell \cdot k_{2}\right)^{2}, P_{34}=\left(\ell \cdot k_{3}\right)^{2}+\left(\ell \cdot k_{4}\right)^{2}$, and $P_{14}=$ $\left(\ell \cdot k_{1}\right)^{2}+\left(\ell \cdot k_{2}\right)^{2}+\left(\ell \cdot k_{3}\right)^{2}+\left(\ell \cdot k_{4}\right)^{2}$. 


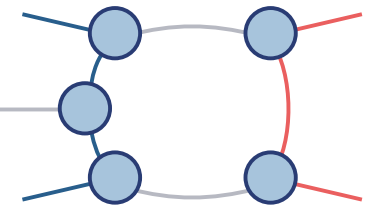

(a) MC1

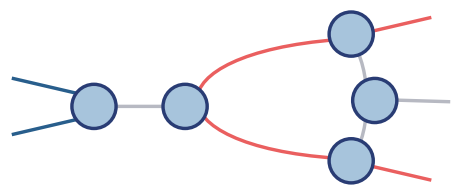

(d) MC4.

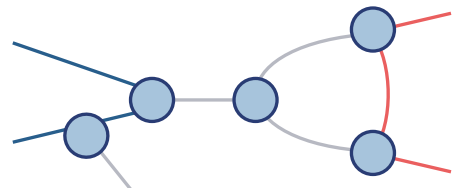

(g) MC7.

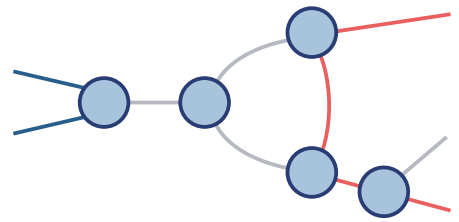

(j) MC10.

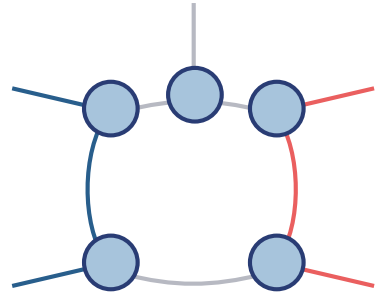

(b) $\mathrm{MC} 2$.

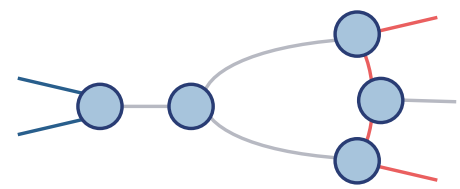

(e) MC5.

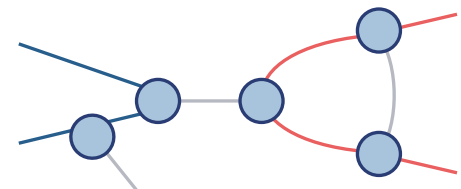

(h) $\mathrm{MC} 8$

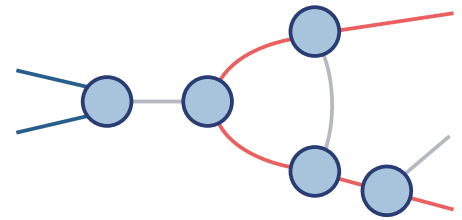

(k) MC11.

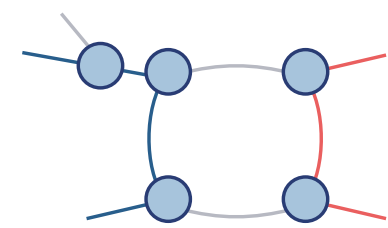

(c) MC3.

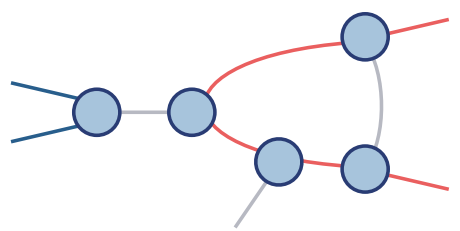

(f) MC6.

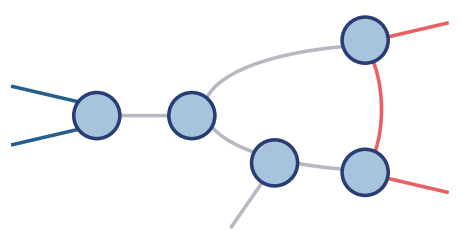

(i) MC9.

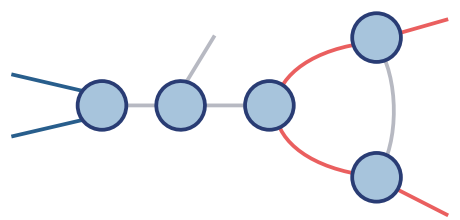

(1) MC12.

Figure 5. Maximal cuts MC1-MC12 of the one-loop five-point amplitude. Light blue blobs represent three-point amplitudes, and exposed legs represent on-shell propagators.

from $\mathrm{N}^{2}$-maximal cuts. However, some delicate cuts must be ignored at $\mathrm{NMC}$ and $\mathrm{N}^{2} \mathrm{MC}$ and the information extracted using $\mathrm{N}^{3} \mathrm{MC}$, as described in the previous section for the four-point amplitude. All $\mathrm{N}^{3}$-max cuts are bubble cuts, and can be found in figure 11. The corrected gravitational numerators can be found in the supplementary material.

\section{Conclusion}

In this paper we demonstrate that the method of maximal cuts in combination with doublecopy can be used to bootstrap graph dressings in Einstein-Hilbert gravity from the double copy of scalar QCD, in a combined procedure we refer to as projective double-copy. Specifically, we systematize the contribution of unitarity cuts of the amplitudes where only gravitational states are allowed to cross the cuts, while allowing the double-copy to provide all relevant gravitational contact terms. Starting with the maximal cuts where all propagators are put on-shell, we work our way up to the necessary $N^{k}$-maximal cuts by systematically releasing cut conditions on propagators. This allows us to project out all non-gravitational 


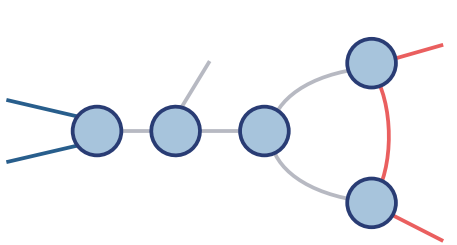

(a) MC13.

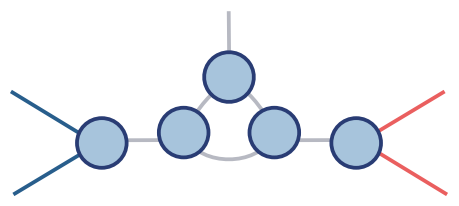

(d) MC16.

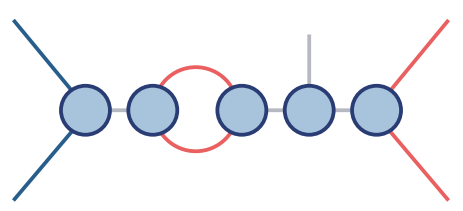

(g) MC19.

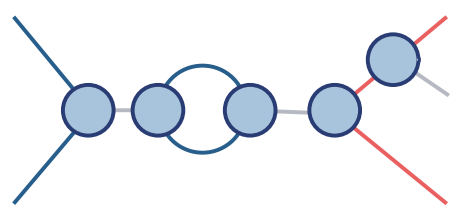

(j) MC22.

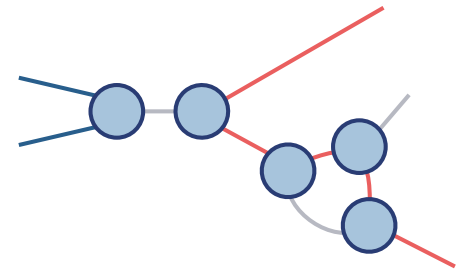

(b) MC14.

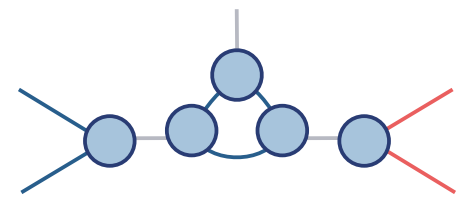

(e) MC17.

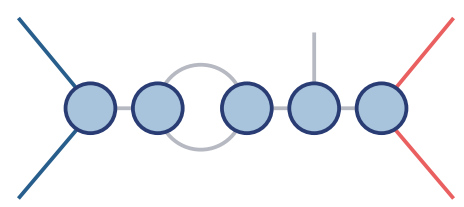

(h) MC20.

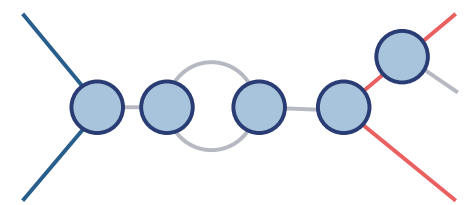

(k) MC23.

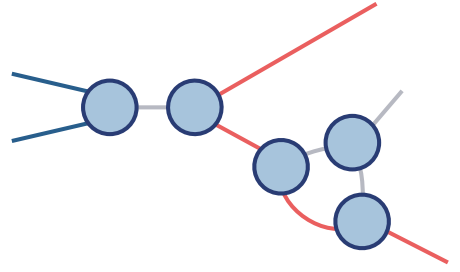

(c) MC15.

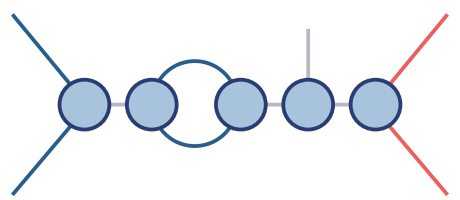

(f) MC18.

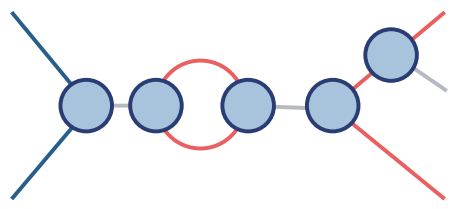

(i) MC21.

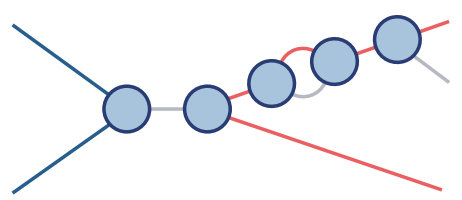

(l) MC24.

Figure 6. Maximal cuts MC13-MC24 of the one-loop five-point amplitude. Light blue blobs represent three-point amplitudes, and exposed legs represent on-shell propagators.

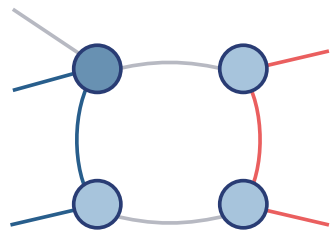

(a) NMC1.

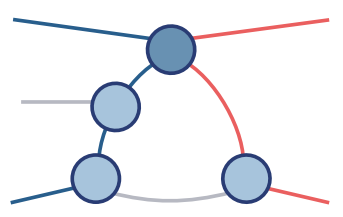

(b) $\mathrm{NMC2}$.

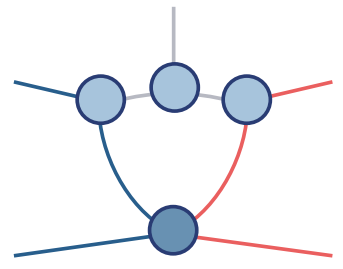

(c) NMC3.

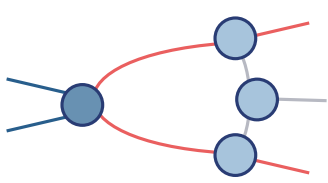

(d) NMC4.

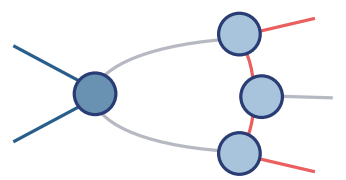

(e) NMC5.

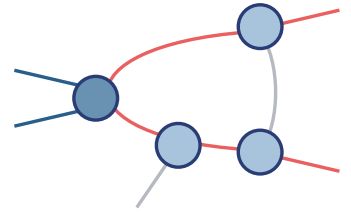

(f) NMC6.

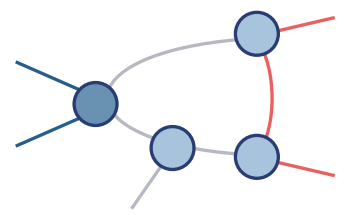

(g) NMC7.

Figure 7. Next-to-maximal box cuts of the one-loop five-point amplitude. Light blue blobs represent three-point amplitudes, darker blobs represent four-point amplitudes, and exposed legs represent on-shell propagators. 


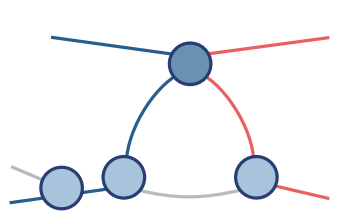

(a) NMC8.

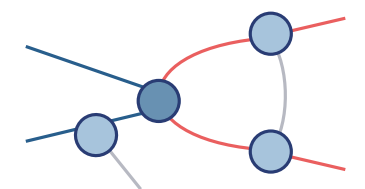

(e) NMC12.

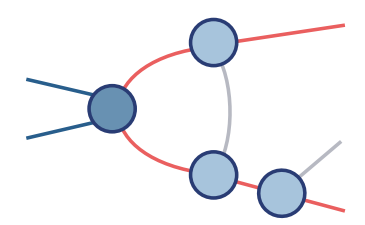

(i) NMC16.

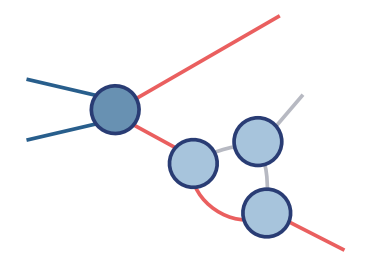

(m) NMC20.

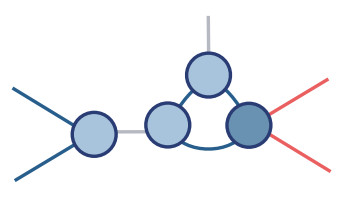

(q) NMC24.

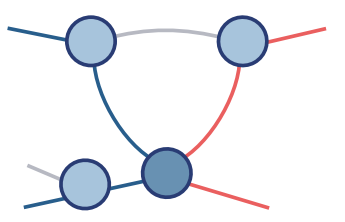

(b) NMC9.

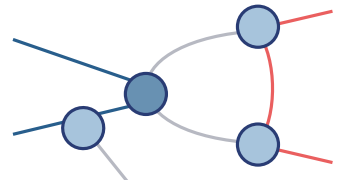

(f) NMC13.

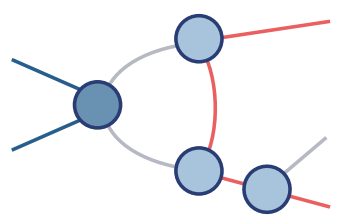

(j) NMC17.

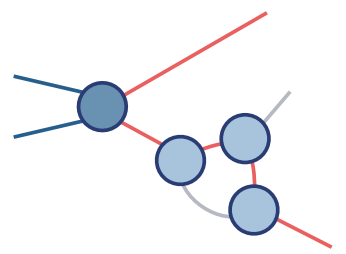

(n) NMC21.

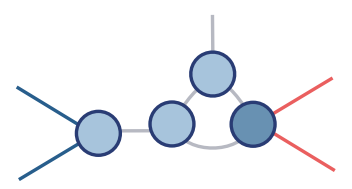

(r) NMC25.

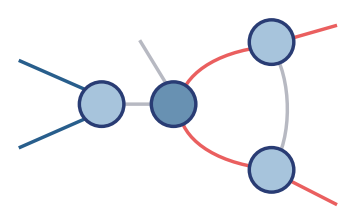

(c) NMC10.

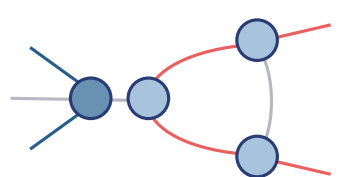

(g) NMC14.

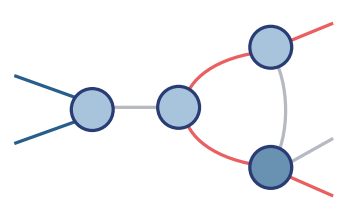

(k) NMC18.

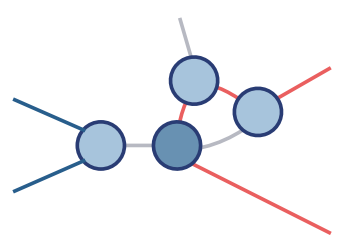

(o) NMC22.

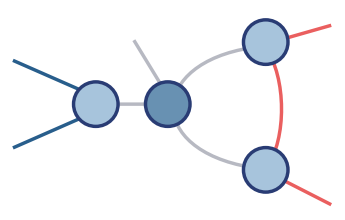

(d) NMC11.

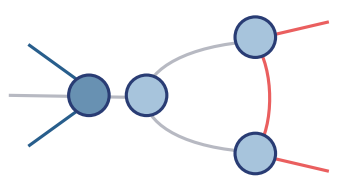

(h) NMC15.

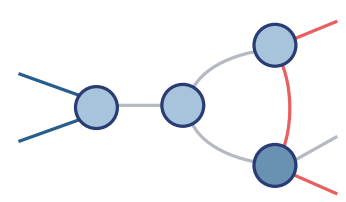

(l) NMC19.

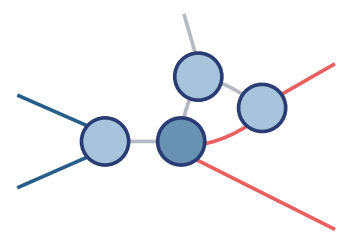

(p) NMC23.

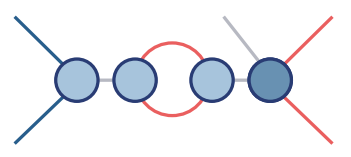

(u) NMC28.

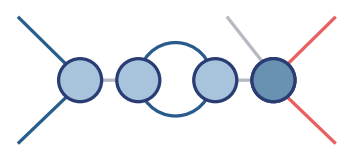

(v) NMC29.

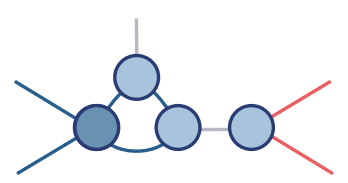

(s) NMC26.

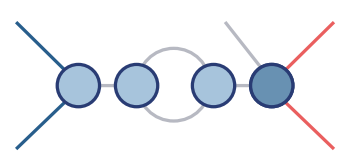

(t) NMC27.

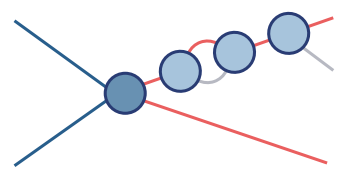

(w) NMC30.

Figure 8. Next-to-maximal cuts of the one-loop five-point amplitude. Light blue blobs represent three-point amplitudes, darker blobs represent four-point amplitudes, and exposed legs represent on-shell propagators. 


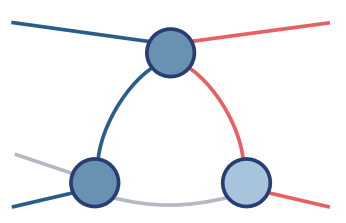

(a) $\mathrm{N}^{2} \mathrm{MC} 1$.

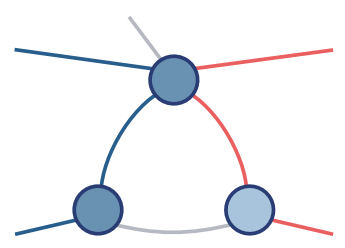

(b) $\mathrm{N}^{2} \mathrm{MC} 2$.

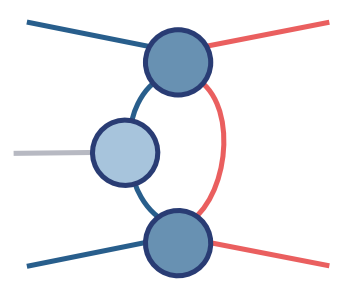

(c) $\mathrm{N}^{2} \mathrm{MC} 3$.

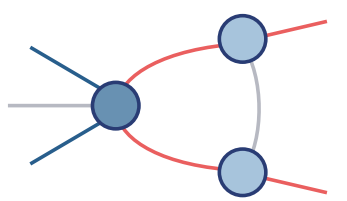

(d) $\mathrm{N}^{2} \mathrm{MC} 4$.

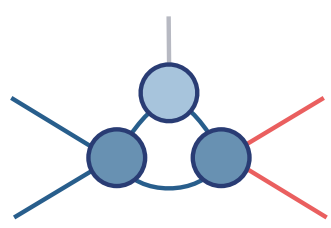

(h) $\mathrm{N}^{2} \mathrm{MC} 8$.

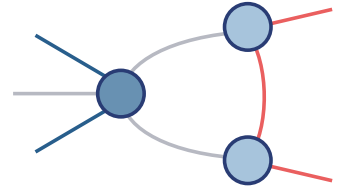

(e) $\mathrm{N}^{2} \mathrm{MC} 5$.

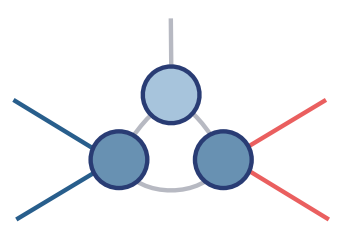

(i) $\mathrm{N}^{2} \mathrm{MC} 9$.

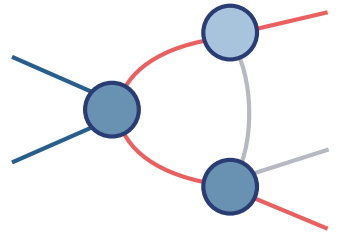

(f) $\mathrm{N}^{2} \mathrm{MC} 6$.

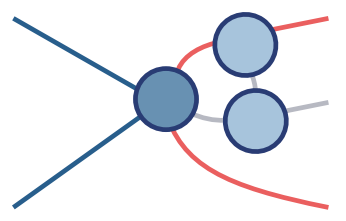

(j) $\mathrm{N}^{2} \mathrm{MC} 10$.

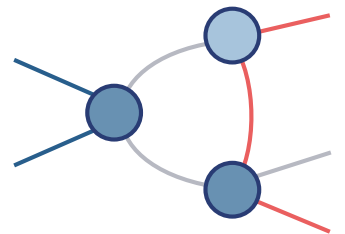

(g) $\mathrm{N}^{2} \mathrm{MC} 7$.

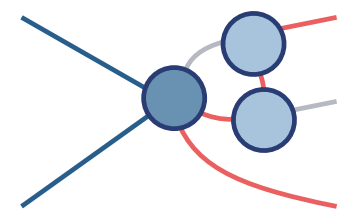

(k) $\mathrm{N}^{2} \mathrm{MC} 11$.

Figure 9. $\mathrm{N}^{2}$-maximal triangle cuts of the one-loop five-point amplitude. Light blue blobs represent three-point amplitudes, darker blobs represent four and five-point amplitudes, and exposed legs represent on-shell propagators.

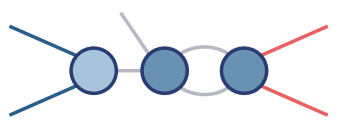

(a) $\mathrm{N}^{2} \mathrm{MC} 12$.

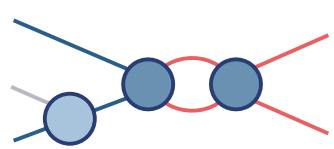

(e) $\mathrm{N}^{2} \mathrm{MC} 16$.

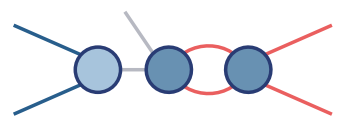

(b) $\mathrm{N}^{2} \mathrm{MC} 13$.

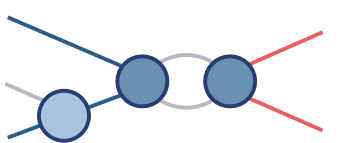

(f) $\mathrm{N}^{2} \mathrm{MC} 17$.

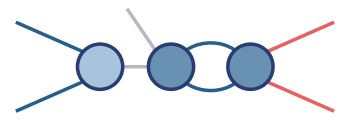

(c) $\mathrm{N}^{2} \mathrm{MC} 14$.

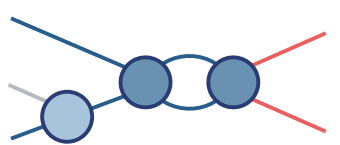

(g) $\mathrm{N}^{2} \mathrm{MC} 18$.

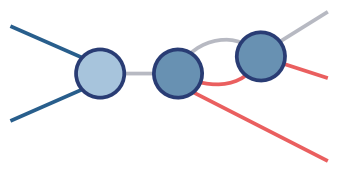

(d) $\mathrm{N}^{2} \mathrm{MC} 15$.

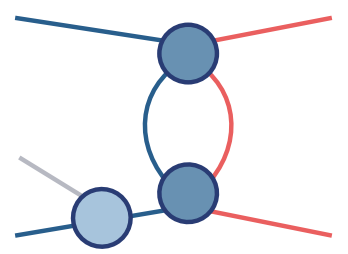

(h) $\mathrm{N}^{2} \mathrm{MC} 19$.

Figure 10. $\mathrm{N}^{2}$-maximal cuts of the one-loop five-point amplitude. Light blue blobs represent three-point amplitudes, darker blobs represent four and five-point amplitudes, and exposed legs represent on-shell propagators. 


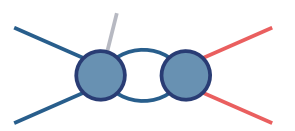

(a) $\mathrm{N}^{3} \mathrm{MC} 1$.

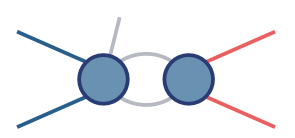

(b) $\mathrm{N}^{3} \mathrm{MC} 2$.

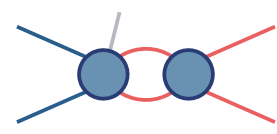

(c) $\mathrm{N}^{3} \mathrm{MC} 3$

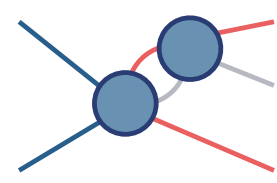

(d) $\mathrm{N}^{3} \mathrm{MC} 4$.

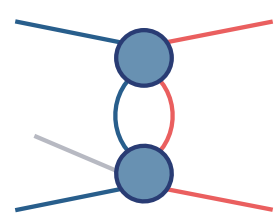

(e) $\mathrm{N}^{3} \mathrm{MC} 5$.

Figure 11. $\mathrm{N}^{3}$-maximal bubble cuts of the one-loop five-point amplitude. Dark blue blobs represent four and five-point amplitudes, and exposed legs represent on-shell propagators.

states from the graph dressings. For tree-level amplitudes with a single massive scalar we find that no correction is necessary up to five-point, while tree- and one-loop amplitudes with more than one massive scalar do contain excess states that are removed. We expect these integrands to be useful to understanding and developing optimized approaches to previously uncalculated classical predictions relevant to gravitational wave astrophysics.

The success and relative simplicity of this procedure promises possible verification for future double copy schemes. It would be intriguing to investigate whether including massless ghostly matter in the original theory in a strategic manner will remove the excess states at loop-level, and the method of maximal cuts can here provide a straight-forward comparison for the resulting amplitudes.

\section{Acknowledgments}

We would like to thank Donal O'Connell for encouragement and detailed discussions regarding classical limits. IVH is supported by the European Union's Horizon 2020 research and innovation programme under the Marie Skłodowska-Curie grant agreement No. 764850 (SAGEX). This work was supported by the DOE under contract DE-SC0021485 and by the Alfred P. Sloan Foundation.

Open Access. This article is distributed under the terms of the Creative Commons Attribution License (CC-BY 4.0), which permits any use, distribution and reproduction in any medium, provided the original author(s) and source are credited.

\section{References}

[1] Z. Bern, J.J.M. Carrasco and H. Johansson, New Relations for Gauge-Theory Amplitudes, Phys. Rev. D 78 (2008) 085011 [arXiv:0805.3993] [InSPIRE].

[2] Z. Bern, J.J.M. Carrasco and H. Johansson, Perturbative Quantum Gravity as a Double Copy of Gauge Theory, Phys. Rev. Lett. 105 (2010) 061602 [arXiv:1004.0476] [InSPIRE].

[3] Z. Bern, J.J.M. Carrasco, M. Chiodaroli, H. Johansson and R. Roiban, The Duality Between Color and Kinematics and its Applications, arXiv:1909.01358 [INSPIRE].

[4] H. Kawai, D.C. Lewellen and S.H.H. Tye, A Relation Between Tree Amplitudes of Closed and Open Strings, Nucl. Phys. B 269 (1986) 1 [InSPIRE]. 
[5] Z. Bern, J.J.M. Carrasco, W.-M. Chen, H. Johansson and R. Roiban, Gravity Amplitudes as Generalized Double Copies of Gauge-Theory Amplitudes, Phys. Rev. Lett. 118 (2017) 181602 [arXiv: 1701.02519] [INSPIRE].

[6] H. Johansson and A. Ochirov, Color-Kinematics Duality for QCD Amplitudes, JHEP 01 (2016) 170 [arXiv: 1507.00332] [INSPIRE].

[7] J. Plefka, C. Shi and T. Wang, Double copy of massive scalar QCD, Phys. Rev. D 101 (2020) 066004 [arXiv:1911.06785] [INSPIRE].

[8] J.J.M. Carrasco and I.A. Vazquez-Holm, Loop-Level Double-Copy for Massive Quantum Particles, Phys. Rev. D 103 (2021) 045002 [arXiv:2010.13435] [INSPIRE].

[9] C. Cheung, I.Z. Rothstein and M.P. Solon, From Scattering Amplitudes to Classical Potentials in the Post-Minkowskian Expansion, Phys. Rev. Lett. 121 (2018) 251101 [arXiv: 1808.02489] [INSPIRE].

[10] D.A. Kosower, B. Maybee and D. O'Connell, Amplitudes, Observables, and Classical Scattering, JHEP 02 (2019) 137 [arXiv: 1811.10950] [INSPIRE].

[11] B. Maybee, D. O'Connell and J. Vines, Observables and amplitudes for spinning particles and black holes, JHEP 12 (2019) 156 [arXiv: 1906.09260] [INSPIRE].

[12] R. Saotome and R. Akhoury, Relationship Between Gravity and Gauge Scattering in the High Energy Limit, JHEP 01 (2013) 123 [arXiv:1210.8111] [INSPIRE].

[13] R. Monteiro, D. O'Connell and C.D. White, Black holes and the double copy, JHEP 12 (2014) 056 [arXiv: 1410.0239] [INSPIRE].

[14] A. Luna, R. Monteiro, D. O'Connell and C.D. White, The classical double copy for Taub-NUT spacetime, Phys. Lett. B $\mathbf{7 5 0}$ (2015) 272 [arXiv: 1507.01869] [INSPIRE].

[15] A.K. Ridgway and M.B. Wise, Static Spherically Symmetric Kerr-Schild Metrics and Implications for the Classical Double Copy, Phys. Rev. D 94 (2016) 044023 [arXiv: 1512.02243] [INSPIRE].

[16] A. Luna, R. Monteiro, I. Nicholson, D. O'Connell and C.D. White, The double copy: Bremsstrahlung and accelerating black holes, JHEP 06 (2016) 023 [arXiv: 1603.05737] [INSPIRE].

[17] C.D. White, Exact solutions for the biadjoint scalar field, Phys. Lett. B 763 (2016) 365 [arXiv: 1606. 04724] [INSPIRE].

[18] W.D. Goldberger and A.K. Ridgway, Radiation and the classical double copy for color charges, Phys. Rev. D 95 (2017) 125010 [arXiv:1611.03493] [INSPIRE].

[19] G. Cardoso, S. Nagy and S. Nampuri, Multi-centered $\mathcal{N}=2$ BPS black holes: a double copy description, JHEP 04 (2017) 037 [arXiv: 1611.04409] [InSPIRE].

[20] A. Luna et al., Perturbative spacetimes from Yang-Mills theory, JHEP 04 (2017) 069 [arXiv: 1611.07508] [INSPIRE].

[21] W.D. Goldberger, S.G. Prabhu and J.O. Thompson, Classical gluon and graviton radiation from the bi-adjoint scalar double copy, Phys. Rev. D 96 (2017) 065009 [arXiv:1705.09263] [INSPIRE].

[22] P.-J. De Smet and C.D. White, Extended solutions for the biadjoint scalar field, Phys. Lett. $B 775$ (2017) 163 [arXiv:1708.01103] [InSPIRE].

[23] N. Bahjat-Abbas, A. Luna and C.D. White, The Kerr-Schild double copy in curved spacetime, JHEP 12 (2017) 004 [arXiv:1710.01953] [INSPIRE]. 
[24] M. Carrillo-González, R. Penco and M. Trodden, The classical double copy in maximally symmetric spacetimes, JHEP 04 (2018) 028 [arXiv:1711.01296] [INSPIRE].

[25] W.D. Goldberger, J. Li and S.G. Prabhu, Spinning particles, axion radiation, and the classical double copy, Phys. Rev. D 97 (2018) 105018 [arXiv:1712.09250] [INSPIRE].

[26] J. Li and S.G. Prabhu, Gravitational radiation from the classical spinning double copy, Phys. Rev. D 97 (2018) 105019 [arXiv: 1803.02405] [INSPIRE].

[27] A. Ilderton, Screw-symmetric gravitational waves: a double copy of the vortex, Phys. Lett. B 782 (2018) 22 [arXiv: 1804.07290] [INSPIRE].

[28] C.-H. Shen, Gravitational Radiation from Color-Kinematics Duality, JHEP 11 (2018) 162 [arXiv: 1806.07388] [INSPIRE].

[29] K. Lee, Kerr-Schild Double Field Theory and Classical Double Copy, JHEP 10 (2018) 027 [arXiv: 1807.08443] [INSPIRE].

[30] J. Plefka, J. Steinhoff and W. Wormsbecher, Effective action of dilaton gravity as the classical double copy of Yang-Mills theory, Phys. Rev. D 99 (2019) 024021 [arXiv: 1807.09859] [INSPIRE].

[31] D.S. Berman, E. Chacón, A. Luna and C.D. White, The self-dual classical double copy, and the Eguchi-Hanson instanton, JHEP 01 (2019) 107 [arXiv: 1809.04063] [INSPIRE].

[32] M. Gurses and B. Tekin, Classical Double Copy: Kerr-Schild-Kundt metrics from Yang-Mills Theory, Phys. Rev. D 98 (2018) 126017 [arXiv:1810.03411] [InSPIRE].

[33] N. Bahjat-Abbas, R. Stark-Muchão and C.D. White, Biadjoint wires, Phys. Lett. B 788 (2019) 274 [arXiv: 1810.08118] [INSPIRE].

[34] A. Luna, R. Monteiro, I. Nicholson and D. O'Connell, Type D Spacetimes and the Weyl Double Copy, Class. Quant. Grav. 36 (2019) 065003 [arXiv:1810.08183] [InSPIRE].

[35] Athira P.V. and A. Manu, Classical double copy from Color Kinematics duality: A proof in the soft limit, Phys. Rev. D 101 (2020) 046014 [arXiv:1907.10021] [INSPIRE].

[36] M. Carrillo González, B. Melcher, K. Ratliff, S. Watson and C.D. White, The classical double copy in three spacetime dimensions, JHEP 07 (2019) 167 [arXiv:1904.11001] [INSPIRE].

[37] Y.-T. Huang, U. Kol and D. O'Connell, Double copy of electric-magnetic duality, Phys. Rev. D 102 (2020) 046005 [arXiv: 1911.06318] [InSPIRE].

[38] R. Alawadhi, D.S. Berman, B. Spence and D. Peinador Veiga, S-duality and the double copy, JHEP 03 (2020) 059 [arXiv:1911.06797] [INSPIRE].

[39] W.T. Emond, Y.-T. Huang, U. Kol, N. Moynihan and D. O'Connell, Amplitudes from Coulomb to Kerr-Taub-NUT, arXiv:2010.07861 [INSPIRE].

[40] D.S. Berman, K. Kim and K. Lee, The classical double copy for M-theory from a Kerr-Schild ansatz for exceptional field theory, JHEP 04 (2021) 071 [arXiv: 2010.08255] [INSPIRE].

[41] T. Adamo, E. Casali, L. Mason and S. Nekovar, Scattering on plane waves and the double copy, Class. Quant. Grav. 35 (2018) 015004 [arXiv:1706.08925] [InSPIRE].

[42] T. Adamo, E. Casali, L. Mason and S. Nekovar, Plane wave backgrounds and colour-kinematics duality, JHEP 02 (2019) 198 [arXiv:1810.05115] [INSPIRE].

[43] Z. Bern, C. Cheung, R. Roiban, C.-H. Shen, M.P. Solon and M. Zeng, Scattering Amplitudes and the Conservative Hamiltonian for Binary Systems at Third Post-Minkowskian Order, Phys. Rev. Lett. 122 (2019) 201603 [arXiv:1901.04424] [INSPIRE]. 
[44] Z. Bern, C. Cheung, R. Roiban, C.-H. Shen, M.P. Solon and M. Zeng, Black Hole Binary Dynamics from the Double Copy and Effective Theory, JHEP 10 (2019) 206 [arXiv: 1908.01493] [INSPIRE].

[45] P.H. Damgaard, K. Haddad and A. Helset, Heavy Black Hole Effective Theory, JHEP 11 (2019) 070 [arXiv: 1908.10308] [INSPIRE].

[46] E. Herrmann, J. Parra-Martinez, M.S. Ruf and M. Zeng, Gravitational Bremsstrahlung from Reverse Unitarity, Phys. Rev. Lett. 126 (2021) 201602 [arXiv:2101.07255] [INSPIRE].

[47] Z. Bern et al., Scattering Amplitudes and Conservative Binary Dynamics at $\mathcal{O}\left(G^{4}\right)$, Phys. Rev. Lett. 126 (2021) 171601 [arXiv:2101.07254] [INSPIRE].

[48] E. Herrmann, J. Parra-Martinez, M.S. Ruf and M. Zeng, Radiative classical gravitational observables at $\mathcal{O}\left(G^{3}\right)$ from scattering amplitudes, JHEP 10 (2021) 148 [arXiv:2104.03957] [INSPIRE].

[49] N.E.J. Bjerrum-Bohr, P.H. Damgaard, L. Planté and P. Vanhove, Classical gravity from loop amplitudes, Phys. Rev. D 104 (2021) 026009 [arXiv:2104.04510] [inSPIRE].

[50] N.E.J. Bjerrum-Bohr, P.H. Damgaard, L. Planté and P. Vanhove, The Amplitude for Classical Gravitational Scattering at Third Post-Minkowskian Order, arXiv:2105.05218 [INSPIRE].

[51] H. Johansson and A. Ochirov, Double copy for massive quantum particles with spin, JHEP 09 (2019) 040 [arXiv: 1906.12292] [INSPIRE].

[52] Y.F. Bautista and A. Guevara, On the Double Copy for Spinning Matter, arXiv:1908.11349 [INSPIRE].

[53] Z. Bern, L.J. Dixon, D.C. Dunbar, M. Perelstein and J.S. Rozowsky, On the relationship between Yang-Mills theory and gravity and its implication for ultraviolet divergences, Nucl. Phys. B 530 (1998) 401 [hep-th/9802162] [INSPIRE].

[54] Z. Bern, D.C. Dunbar and T. Shimada, String based methods in perturbative gravity, Phys. Lett. B 312 (1993) 277 [hep-th/9307001] [INSPIRE].

[55] D.C. Dunbar and P.S. Norridge, Calculation of graviton scattering amplitudes using string based methods, Nucl. Phys. B 433 (1995) 181 [hep-th/9408014] [INSPIRE].

[56] Z. Bern, J.J.M. Carrasco, H. Johansson and D.A. Kosower, Maximally supersymmetric planar Yang-Mills amplitudes at five loops, Phys. Rev. D 76 (2007) 125020 [arXiv: 0705.1864] [INSPIRE].

[57] H. Johansson and A. Ochirov, Pure Gravities via Color-Kinematics Duality for Fundamental Matter, JHEP 11 (2015) 046 [arXiv:1407.4772] [INSPIRE].

[58] A. Luna, I. Nicholson, D. O'Connell and C.D. White, Inelastic Black Hole Scattering from Charged Scalar Amplitudes, JHEP 03 (2018) 044 [arXiv: 1711.03901] [INSPIRE].

[59] D.-p. Zhu, Zeros in Scattering Amplitudes and the Structure of NonAbelian Gauge Theories, Phys. Rev. D 22 (1980) 2266 [InSPIRE].

[60] C.J. Goebel, F. Halzen and J.P. Leveille, Angular zeros of Brown, Mikaelian, Sahdev, and Samuel and the factorization of tree amplitudes in gauge theories, Phys. Rev. D 23 (1981) 2682 [INSPIRE].

[61] L.A. Harland-Lang, Planar radiation zeros in five-parton QCD amplitudes, JHEP 05 (2015) 146 [arXiv: 1503.06798] [INSPIRE]. 
[62] R. Britto, F. Cachazo, B. Feng and E. Witten, Direct proof of tree-level recursion relation in Yang-Mills theory, Phys. Rev. Lett. 94 (2005) 181602 [hep-th/0501052] [InSPIRE].

[63] P. Benincasa, C. Boucher-Veronneau and F. Cachazo, Taming Tree Amplitudes In General Relativity, JHEP 11 (2007) 057 [hep-th/0702032] [INSPIRE].

[64] Z. Bern, J.J.M. Carrasco, L.J. Dixon, H. Johansson and R. Roiban, Simplifying Multiloop Integrands and Ultraviolet Divergences of Gauge Theory and Gravity Amplitudes, Phys. Rev. D 85 (2012) 105014 [arXiv: 1201.5366] [INSPIRE].

[65] Z. Bern and A.G. Morgan, Massive loop amplitudes from unitarity, Nucl. Phys. B 467 (1996) 479 [hep-ph/9511336] [INSPIRE]. 